\title{
Simple Mechanisms for a Subadditive Buyer and Applications to Revenue Monotonicity
}

\author{
AVIAD RUBINSTEIN, UC Berkeley \\ S. MATTHEW WEINBERG, Princeton University
}

\begin{abstract}
We study the revenue maximization problem of a seller with $n$ heterogeneous items for sale to a single buyer whose valuation function for sets of items is unknown and drawn from some distribution $D$. We show that if $D$ is a distribution over subadditive valuations with independent items, then the better of pricing each item separately or pricing only the grand bundle achieves a constant-factor approximation to the revenue of the optimal mechanism. This includes buyers who are $k$-demand, additive up to a matroid constraint, or additive up to constraints of any downward-closed set system (and whose values for the individual items are sampled independently), as well as buyers who are fractionally subadditive with item multipliers drawn independently. Our proof makes use of the core-tail decomposition framework developed in prior work showing similar results for the significantly simpler class of additive buyers.

In the second part of the article, we develop a connection between approximately optimal simple mechanisms and approximate revenue monotonicity with respect to buyers' valuations. Revenue non-monotonicity is the phenomenon that sometimes strictly increasing buyers' values for every set can strictly decrease the revenue of the optimal mechanism. Using our main result, we derive a bound on how bad this degradation can be (and dub such a bound a proof of approximate revenue monotonicity); we further show that better bounds on approximate monotonicity imply a better analysis of our simple mechanisms.
\end{abstract}

CCS Concepts: • Theory of computation $\rightarrow$ Algorithmic game theory and mechanism design; Algorithmic mechanism design; Computational pricing and auctions;

Additional Key Words and Phrases: Revenue optimization, combinatorial valuations, simple auctions, revenue monotonicity

ACM Reference format:

Aviad Rubinstein and S. Matthew Weinberg. 2018. Simple Mechanisms for a Subadditive Buyer and Applications to Revenue Monotonicity. ACM Trans. Econ. Comput. 6, 3-4, Article 19 (October 2018), 25 pages.

https://doi.org/10.1145/3105448

\section{INTRODUCTION}

Consider a revenue-maximizing seller with $n$ heterogeneous items for sale to a single buyer whose value for sets of items is unknown, but drawn from a known distribution $D$. When $n=1$, seminal work of Myerson (1981) and Riley and Zeckhauser (1983) shows that the optimal

This research was supported by NSF grants CCF0964033 and CCF1408635, and by Templeton Foundation grant 3966. This work was done in part at the Simons Institute for the Theory of Computing and while visiting Princeton University.

Authors' addresses: A. Rubinstein, Department of Electrical Engineering and Computer Science, UC Berkeley, 2000 Carlton Street, Berkeley CA 94720; email: aviad@eecs.berkeley.edu; S. M. Weinberg, Department of Computer Science, Princeton University, 35 Olden Street, Princeton, NJ 08540; email: smweinberg@princeton.edu.

Permission to make digital or hard copies of all or part of this work for personal or classroom use is granted without fee provided that copies are not made or distributed for profit or commercial advantage and that copies bear this notice and the full citation on the first page. Copyrights for components of this work owned by others than ACM must be honored. Abstracting with credit is permitted. To copy otherwise, or republish, to post on servers or to redistribute to lists, requires prior specific permission and/or a fee. Request permissions from permissions@acm.org.

(C) 2018 Association for Computing Machinery.

2167-8375/2018/10-ART19 \$15.00

https://doi.org/10.1145/3105448 
selling scheme simply sets the price $p^{*}=\arg \max \{p \cdot \operatorname{Pr}[v \geq p \mid v \leftarrow D]\}$. Thirty years later, understanding the structure of the optimal mechanism when $n>1$ still remains a central open problem. Unfortunately, it is well-known that the optimal mechanism may require randomization, behave non-monotonically, and be computationally hard to find, even in very simple instances (Thanassoulis 2004; Pavlov 2011; Briest et al. 2010; Daskalakis et al. 2014; Chen et al. 2014; Hart and Nisan 2013; Hart and Reny 2012; Papadimitriou et al. 2016). In light of this, recent work began studying the performance of especially simple auctions through the lens of approximation. Remarkably, these works have shown that when the bidder's valuation is additive, ${ }^{1}$ and her value for each item is drawn independently, very simple mechanisms can achieve quite good approximation ratios. Specifically, techniques developed in this series of works proves that the better of setting Myerson's reserve on each item separately or setting Myerson's reserve on the grand bundle of all items together achieves a 6-approximation (Hart and Nisan 2012; Li and Yao 2013; Babaioff et al. 2014).

While this model of buyer values is certainly mathematically interesting and economically motivated, it is also perhaps too simplistic to have broad real-world applications. A central question left open by these works is whether or not simple mechanisms can still approximate optimal ones in more general settings. In this article, we resolve this question in the affirmative, showing that the better of selling separately (we will henceforth use SREv to denote the revenue of the optimal such mechanism) or together (henceforth BREv) still obtains a constant-factor approximation to the optimal revenue (henceforth REv) when buyer values are combinatorial in nature but complement-free.

Informal Theorem 1. Let $D$ be any distribution over subadditive valuation functions with independent items. Then $\max \{\mathrm{SREv}, \mathrm{BREv}\} \geq \Omega(1) \cdot \mathrm{Rev}$. Furthermore, prices providing this guarantee can be found computationally efficiently.

We postpone a formal definition of exactly what it means for $D$ to have "independent items" to Section 2. We note here a few instantiations of our model in commonly studied settings (from least to most general):

$-k$-demand: The buyer has value $v_{i}$ for item $i$, and the $v_{i}$ s are drawn independently. The buyer's value for a set $S$ is $v(S)=\max _{T \subseteq S,|T| \leq k}\left\{\sum_{i \in T} v_{i}\right\}$.

- Additive up to constraints $\mathcal{I}: \mathcal{I}$ is some downward-closed set system on $[n]$. The buyer has value $v_{i}$ for item $i$, and the $v_{i}$ s are drawn independently. $v(S)=\max _{T \subseteq S, T \in I}\left\{\sum_{i \in T} v_{i}\right\}$.

-Fractionally subadditive: buyer has "possible values" $\left\{v_{i j}\right\}_{j}$ for item $i$, and the sets $\left\{v_{i j}\right\}_{j}$ are drawn independently across items (but may be correlated within an item). $v(S)=$ $\max _{j}\left\{\sum_{i \in S} v_{i j}\right\}$.

A recent book of Hartline (2011) provides a fantastic discussion of the role of approximation in mechanism design. Before proceeding, it is worth repeating some aspects of this discussion to view our result in the proper context. One should not interpret our main result as claiming that sellers should be satisfied with a constant fraction of the optimal obtainable revenue, but rather as studying the tradeoff between simplicity and optimality. Sometimes, the optimal mechanism simply isn't an option: perhaps it is prohibitively complex to implement, prohibitively frustrating for buyers to participate, or prohibitively difficult (computationally) to find. And even when the optimal mechanism is a feasible option, the desire for simplicity and transparency may outweigh the expected loss in revenue. Similarly, one should not interpret the ratios obtained in our main result (they are noticeably larger than 6) as ratios that one might expect to trade off in practice, as these are provable bounds for worst-case instances.

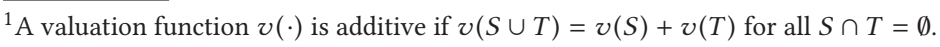




\subsection{Challenges of Combinatorial Valuations}

The design of simple, approximately optimal mechanisms for any non-trivial multi-item setting has been a large focus for much of the algorithmic game theory community over the past decade. Even "simple" settings with additive or unit-demand valuations required significant breakthroughs. The key insight enabling these breakthroughs for additive buyers is that the buyer's valuation is separable across items. While the optimal mechanism can still be quite bizarre despite this realization (Hart and Reny 2012), this fact enables certain elementary decomposition theorems that are surprisingly powerful (e.g., the "Marginal Mechanism" (Cai and Huang 2013; Hart and Nisan 2012)). However, these theorems are extremely sensitive to being able to separate the marginal contribution of different items exactly (and not just via upper/lower bounds). This is due to the phenomenon that a slight miscalculation in estimating a buyer's value may cause her to change preferences entirely, resulting in a potentially unbounded loss of revenue. One of our main technical contributions is overcoming this obstacle by providing an approximate version of these decomposition theorems.

A further complication in applying these previous techniques is that they all make use of the fact that $\operatorname{SREv}\left(D_{1} \times \cdots \times D_{n}\right)=\sum_{i} \operatorname{SREv}\left(D_{i}\right)$. This claim is not even approximately true for subadditive buyers, and the ratio between the two values could be as large as $n$ (the right-hand side is always larger). To have any hope of applying these tools, we therefore need a proxy for SREv that at least approximately has this separability property.

For unit-demand buyers, the key insight behind the mechanisms designed in Chawla et al. (2007, 2010a, 2010b) and Kleinberg and Weinberg (2012) is that every multi-dimensional problem instance has a related single-dimensional problem instance, and there is a correspondence between truthful mechanisms in the two instances. This realization means that one can instead design mechanisms for the single-dimensional setting, where optimal mechanisms are well understood due to Myerson's virtual values, and translate them in a black-box manner to mechanisms for the original instance. While these techniques have proven extremely fruitful in the design of mechanisms for multiple unit-demand buyers and sophisticated feasibility constraints, they have also proven to be limited in use to unit-demand settings. A special case of our results can be seen as providing an alternative proof of the single-buyer result of Chawla et al. (2007) (albeit with a significantly worse constant) that doesn't require virtual valuation machinery.

Aside from the difficulties in applying existing machinery to design optimal mechanisms for combinatorial valuations, formal barriers exist as well. For instance, it is a trivial procedure for an additive buyer to select his utility-maximizing set of items when facing an item-pricing, and finding the revenue-optimal item-pricing is also trivial (just find the optimal price for each item separately). Yet for a subadditive buyer, both tasks are quite non-trivial. Just computing the expected revenue obtained by a fixed item-pricing is NP-hard. Worse, the buyer's problem of just selecting her utility-maximizing set from a given item-pricing is also NP-hard! Therefore, buyers may behave quite unpredictably in the face of an item-pricing depending on how well they can optimize. Moreover, even if we are willing to assume that the buyer has the computational power to select her utility-maximizing set, it is known still that (without our independence assumption) finding an $n^{c}$-approximately optimal mechanism is NP-hard for all $c=O(1)$ (Cai et al. 2013). We sidestep all these difficulties by not attempting to compute or approximate SREv at all, nor trying to predict bizarre buyer behavior. We instead perform our analysis on revenue contributions only of items purchased when the buyer is not willing to purchase any others. Buyer behavior in such instances is predictable and easily computable: simply purchase the unique item for which $v(\{i\})>p_{i}$. It is surprising that such an analysis suffices, as it completely ignores any revenue contribution coming from the entirely plausible event that the buyer is willing to purchase multiple items. 


\subsection{Techniques}

We prove our main theorem by making use of the core-tail decomposition framework introduced by $\mathrm{Li}$ and Yao (2013). There are three high-level steps to applying the framework. The first is proving a "core decomposition" lemma that separates the optimal revenue into contributions from items that the buyer values very highly (the "tail"), and items that the buyer values not so high (the "core"). The second is showing that the contribution from the tail can be approximated well by SREv. The third is showing that the contribution from the core can be approximated well by $\max \{$ SREv, BREv $\}$.

The Core Decomposition Lemma. The proof of the original Core Decomposition Lemma in Li and Yao (2013) was obtained by cleverly stringing together simple claims proved in Hart and Nisan (2012). As discussed above, these seemingly "obvious" claims may not extend beyond additive valuations over independent items, due to the fact that the buyer's value cannot be separated across items. Nevertheless, we are able to prove an approximate version of the core decomposition lemma for subadditive buyers (Lemma 3.6) by making use of ideas from reductions from $\epsilon$-truthful mechanisms to fully truthful ones. Like in Babaioff et al. (2014), our core decomposition lemma holds for many buyers. The proof for a single buyer, which is the focus of this article, can be found in Section 3.1. We also provide (Section 5) a more technically involved proof for many buyers, which builds on heavier tools from Bei and Huang (2011), Hartline et al. (2011), and Daskalakis and Weinberg (2012).

Bounding the Tail's Contribution. Arguments for bounding the contribution from the tail in prior work (and ours) use the following reasoning. If the cutoff between core and tail is sufficiently high, then the probability that $k$ items are simultaneously in the tail for a sampled valuation decays exponentially in $k$. If one can also show that the approximation guarantee of SREV decays subexponentially in $k$, then we can bound the gap between SREv and the tail's contribution by a constant factor. We show that indeed the approximation guarantee of SREV decays only polynomially in $k$.

Bounding the Core's Contribution. Arguments for bounding the contribution from the core in prior work (and ours) use the following reasoning. The total expected value for items in the core is a subadditive function of independent random variables (bounded above by the core-tail cutoff). If the cutoff between core and tail is sufficiently low, then one of two things must happen. Either the expected contribution from the core is also small, in which case SREv itself provides a good approximation, or the expected contribution is large, and therefore also large with respect to the cutoffs. In the latter case, a concentration bound implies that BREV must provide a good approximation. In the additive case, the appropriate concentration bound is Chebyshev's inequality. In the subadditive case, we need heavier tools, and apply a concentration bound due to Schechtman (2003).

\subsection{Connection to Approximate Revenue-Monotonicity}

Consider designing revenue-optimal mechanisms for two different markets, and suppose that the valuations of the consumers in the first market first-order stochastically dominate ${ }^{2}$ the valuations of the consumers in the second market. It then seems reasonable to expect that the optimal revenue achieved from the first market, $\operatorname{Rev}\left(D^{+}\right)$, should be at least as large as the revenue achieved from the dominated market, $\operatorname{Rev}(D)$. When there is just a single item for sale, this is an easy corollary of the format for Myerson's optimal auction. Yet Hart and Reny provided an example where this intuition breaks even in a setting as simple as an additive buyer with i.i.d. values for two items (Hart and Reny 2012). Surprisingly, their example shows that it is possible to make strictly more revenue

\footnotetext{
${ }^{2}$ We say that a distribution $D^{+}$over valuation functions $v^{+}$first-order stochastically dominates distribution $D$ over valuation functions $v$ if the probability spaces can be coupled so that for every subset $S, v^{+}(S) \geq v(S)$.
} 
in a market when buyers have strictly less value for your goods, and the market need not even be very complex for this phenomenon to occur.

A natural question to ask then, is how large this anomaly can be. For example, Hart and Reny's constructions exhibit a (multiplicative) gap of 33/32 between $\operatorname{Rev}\left(D^{+}\right)$and $\operatorname{Rev}(D)$ for an additive buyer with correlated values for two items, and $\left(1+\frac{1}{7000000}\right)$ for an additive buyer with i.i.d. values for two items. Interestingly, the simple mechanisms of Hart and Nisan (2012), Li and Yao (2013), and Babaioff et al. (2014) upper bound the possible gap of any instance where their results apply, since $\operatorname{SREV}$ and BREv are monotone for additive buyers (i.e., $\operatorname{SREv}\left(D^{+}\right) \geq \operatorname{SREv}(D)$ and $\left.\operatorname{BRev}\left(D^{+}\right) \geq \operatorname{BREv}(D)\right)$. Specifically, for an additive buyer the gap is at most $(1+1 / e)$ for two i.i.d. items, 2 for two asymmetric independent items, and 6 for any number of independent items. In Section 4 we show that as a corollary of our results, the gap is also constant for a subadditive buyer with independent items. Interestingly, this connection between approximately optimal simple mechanisms and approximate revenue-monotonicity is also fruitful in the other direction: it turns out that improving the bound on approximate monotonicity for a subadditive buyer would also improve the constant in our main theorem. Finally, we show in Section 4.3 that for an additive buyer with correlated values for two items, the gap is potentially infinite. (This is the case for which Hart and Reny provide a gap of 33/32.) The proof is by a black-box reduction from an example due to Hart and Nisan (2013) that exhibits a similar gap between simple and optimal mechanisms, further demonstrating the connection between these two important research directions.

\subsection{Discussion, Related Work, and Open Problems}

Our work contributes to the recent growing literature on simple, approximately optimal mechanisms. We extend greatly beyond prior work, providing the first simple and approximately optimal mechanisms for buyers with combinatorial valuations. Prior to our work, virtually nothing was known about this setting (modulo the impossibility result of Cai et al. (2013)). Our results also demonstrate the strength of the core-tail decomposition framework developed by Li and Yao to go beyond additive buyers.

In our opinion, the most exciting open question in this area is extending these results to multiple buyers. A beautiful lookahead reduction was previously developed by Yao (2015) for additive buyers, but applying his techniques for combinatorial bidders proved quite challenging. Following a preliminary version of this article, others have built upon our techniques and made quite significant progress on this problem (Cai et al. 2016; Chawla and Miller 2016; Cai and Zhao 2017). To put them in context with respect to ours:

- Cai et al. (2016) provide an alternative proof of Yao's result and improve the constant factor (from 69 to 8 ). They also provide a duality framework, and show that, essentially, all bounds on the optimal revenue used in prior works can be derived by essentially the same dual solution. The only exception we are aware of is our "approximate marginal mechanism" lemma, which to date has no "duality proof" in their framework. It would be interesting to understand this lemma in terms of duality, but not essential for its application.

- Chawla and Miller (2016) provide the first approximately optimal mechanism for multiple combinatorial buyers. Without getting into a full definition, their results apply to a special case of valuations that are "gross substitutes over independent items." They also improve the constant factor in our analysis. Their work did make use of part of our analysis, but required substantial conceptual novelty.

- Most recently, Cai and Zhao (2017) extended their result to multiple buyers that are "XOS over independent items." Their result draws on numerous previous works, those referenced above (Yao 2015; Cai et al. 2016; Chawla and Miller 2016), ours, and also the posted-price 
mechanism of Feldman et al. (2015) for welfare-maximization in Bayesian settings with multiple XOS bidders. They obtain a logarithmic approximation for multiple buyers that are subadditive over independent items, and the main bottleneck in extending their constantfactor approximation from XOS to subadditive is that Feldman et al.'s welfare guarantee only holds for XOS valuations. Obtaining a constant-factor approximation for many buyers with subadditve valuations remains a key open question, although each of the aforementioned articles makes substantial progress beyond ours.

Another important direction is extending our understanding of simple mechanisms to models of limited correlation over values for disjoint sets of items. ${ }^{3}$ Recent independent work of Bateni et al. (2015) addresses this direction, providing approximation guarantees on $\max \{\mathrm{SREV}, \mathrm{BREV}\}$ vs. REv for an additive buyer whose values for items are drawn from a common-base-value distribution and various extensions. Their results also make use of a core-tail decomposition, but the tools they develop beyond the decomposition are disjoint from ours. A natural question in this direction is whether our results extend to settings where buyer values are both combinatorial and exhibit limited correlation between disjoint sets of items, as the end goal is to have a model that encompasses as many real-world instances as possible.

Other related works include an approximately optimal mechanism for an additive buyer when the seller incurs production costs (Ma and Simchi-Levi 2015), and an approximately optimal mechanism for a buyer with restricted complementarities (Eden et al. 2017). Rubinstein (2016) considers a related algorithmic problem of finding the optimal partition mechanism (where the seller posts a take-it-or-leave-it price on disjoint bundles). It remains unknown whether the optimal partition mechanism can provide provably better guarantees than $\max \{\mathrm{SREV}, \mathrm{BREV}\}$ with respect to REv in any domain.

If we were to identify the contributions of the present work that seem to have been most useful in follow-up works, they would be:

- The "approximate marginal mechanism lemma," Lemmas 3.2 and 5.1, have been used (e.g., by Cai and Zhao) to reduce from subadditive/gross substitutes/and so on over independent items to "additive subject to downwards-closed/matroid/etc. constraints with independent items" at the cost of a constant factor.

- Our application of Schechtman's bound in the core, Corollary 3.11, has been used in essentially every follow-up work discussing optimal mechanisms for combinatorial buyers (Chawla and Miller 2016; Cai and Zhao 2017; Eden et al. 2017).

- In the face of buyers for whom selecting a utility-maximizing bundle might be NP-hard, the idea to count revenue only when it is trivial to select a utility-maximizing bundle (e.g., because it is a singleton set) has proven useful in follow-up works such as Eden et al. (2017).

- Moreover, just a meaningful definition of "subadditive over independent items" seemed to be useful in all of the referenced works, and even for the design of combinatorial prophet inequalities (where again some notion of independent is necessary to subvert horrible lower bounds even in the additive case) (Rubinstein and Singla 2017).

\section{PRELIMINARIES}

We focus the initial exposition on the single-buyer problem, and postpone all details regarding auctions for multiple buyers to Section 5. There is a single revenue-maximizing seller with $n$ items for sale to a single buyer. The buyer has combinatorial valuations for the items (i.e., value $v(S)$

\footnotetext{
${ }^{3}$ Note that, as we mention in the previous section, for arbitrary correlated items, the gap can be infinite (Hart and Nisan 2013; Briest et al. 2010).
} 
for receiving set $S$ ), and is quasi-linear and risk-neutral. That is, the buyer's utility for a randomized outcome that awards him set $S$ with probability $A(S)$ while paying (expected) price $p$ is $\sum_{S} A(S) v(S)-p$. The valuation $v(\cdot)$ is unknown to the seller, who has a prior $D$ over possible buyer valuations that is subadditive over independent items, a term we describe below. By the taxation principle, the seller may restrict attention to only lottery systems. In other words, the seller provides a list of potential lotteries (distributions over sets) each with a price, and the buyer chooses the utility-maximizing option.

\subsection{Subadditive Valuations over Independent Items}

We now carefully define what we mean by subadditive valuations over independent items. Intuitively, our model is such that the buyer has some private information $x_{i}$ pertaining to item $i{ }^{4}$ and $D^{\vec{x}}$ is a product distribution over $\mathbb{R}^{n}$ representing the seller's prior over the private information possessed by the buyer. The buyer's valuation for set $S$ is parametrized by the private information she has about items in that set, and can be written as $V\left(\left\langle x_{i}\right\rangle_{i \in S}, S\right)$. In economic terms, this models that the items not received by the buyer impose no externalities. We capture this formally in the definition below:

Definition 2.1. We say that a distribution $D$ over valuation functions $v(\cdot):\{0,1\}^{n} \rightarrow \mathbb{R}$ is subadditive over independent items if:

(1) All $v(\cdot)$ in the support of $D$ exhibit no externalities.

Formally, let $\Omega_{S}=\times_{i \in S} \Omega_{i}$, where each $\Omega_{i}$ is a compact subset of a normed space. There exists a distribution $D^{\vec{x}}$ over $\Omega_{[n]}$ and functions $V_{S}: \Omega_{S} \rightarrow \mathbb{R}$ such that $D$ is the distribution that first samples $\vec{x} \leftarrow D^{\vec{x}}$ and outputs the valuation function $v(\cdot)$ with $v(S)=V_{S}\left(\left\langle x_{i}\right\rangle_{i \in S}\right)$ for all $S$.

(2) All $v(\cdot)$ in the support of $D$ are monotone. That is, $v(S) \leq v(S \cup T)$ for all $S, T$.

(3) All $v(\cdot)$ in the support of $D$ are subadditive. That is, $v(S \cup T) \leq v(S)+v(T)$.

(4) The private information is independent across items. That is, the $D^{\vec{x}}$ guaranteed in property 1 is a product distribution.

We describe now how to encode commonly studied valuation distributions in this model.

Example 2.2. The following types of distributions can be modeled as subadditive over independent items. (Recall that $\vec{x}$ is the vector of independently sampled attributes in the definition above.)

(1) Additive: Let $\Omega_{i}=[0,1]$ and interpret $x_{i}$ as the buyer's value for item $i . V_{S}\left(\left\langle x_{i}\right\rangle_{i \in S}\right)=$ $\sum_{i \in S} x_{i}$

(2) $k$-demand: Let $\Omega_{i}=[0,1]$ and interpret $x_{i}$ as the buyer's value for item $i \cdot V_{S}\left(\left\langle x_{i}\right\rangle_{i \in S}\right)=$ $\max _{T \subseteq S,|T| \leq k}\left\{\sum_{i \in T} x_{i}\right\}$.

(3) Additive up to $\mathcal{I}$ : Let $\Omega_{i}=[0,1]$ and interpret $x_{i}$ as the buyer's value for item $i$. $V_{S}\left(\left\langle x_{i}\right\rangle_{i \in S}\right)=\max _{T \subseteq S, T \in I}\left\{\sum_{i \in T} x_{i}\right\}$

(4) Fractionally subadditive: Let $\Omega_{i}=[0,1]^{k}$ for any finite $k$ and interpret $x_{i}$ as encoding the values $\left\{v_{i j}\right\}_{j \in[k]} . V_{S}\left(\left\langle x_{i}\right\rangle_{i \in S}\right)=\max _{j}\left\{\sum_{i \in S} v_{i j}\right\}$.

\subsection{Notation}

Definition 2.3. For any distribution $D$ of buyer's valuation, we use the following notation, most of which is due to Hart and Nisan (2012) and Babaioff et al. (2014):

\footnotetext{
${ }^{4}$ Think of this information as "information about the buyer's preferences related to item."
} 
$-D_{i}$ : The distribution of $v(\{i\})$ when $v(\cdot) \leftarrow D$.

$-t$ : The cutoff between core and tail. If $v(\{i\})>t$, we say that item $i$ is in the tail. Otherwise it is in the core.

$-D_{A}$ : The distribution $D$, conditioned on $A$ being exactly the set of items in the tail.

$-D_{A}^{T}$ : The distribution $D_{A}$ restricted just to items in the tail (i.e., $A$ ).

$-D_{A}^{C}$ : The distribution $D_{A}$ restricted just to items in the core (i.e., $\bar{A}$ ).

$-p_{i}$ : The probability that element $i$ is in the tail.

$-p_{A}$ : The probability that $A$ is exactly the set of items in the tail (note that $p_{\{i\}} \neq p_{i}$ ).

$-\operatorname{Rev}(D)$ : The maximum revenue obtainable via a truthful mechanism from a buyer with valuation profile drawn from $D$.

$-\operatorname{BRev}(D)$ : The revenue obtainable from a buyer with valuation profile drawn from $D$ by auctioning the grand bundle via Myerson's optimal auction.

$-\operatorname{SREv}(D)$ : The maximum revenue obtainable from a buyer with valuation profile drawn from $D$ by pricing each item separately. Note that when the buyer is not additive, $\operatorname{SREv}(D)$ behaves erratically and is NP-hard to find (Chen et al. 2014).

$-\operatorname{Rev}_{q}(D)$ : For a one-dimensional distribution $D$, the optimal revenue obtained by a reserve price that sells with probability at most $q$.

$-\operatorname{SREv}_{\vec{q}}^{*}(D): \prod_{i}\left(1-q_{i}\right) \cdot \sum_{i} \operatorname{Rev}_{q_{i}}\left(D_{i}\right)$ : a proxy for $\operatorname{SREv}(D)$ that behaves nicer and is easy to compute.

$-\operatorname{VAL}(D)$ : the buyer's expected valuation for the grand bundle, $\mathrm{E}_{v \leftarrow D}[v([n])]$.

When the distribution is clear from the context, we simply use REv, VAL, and so on. Most of this notation is standard following Hart and Nisan (2012), with the exception of $\operatorname{Rev}_{q}$ and $\operatorname{SREV}_{\vec{q}}^{*}$. We introduce $\mathrm{SREV}_{\vec{q}}^{*}$ because it will serve as a proxy to $\mathrm{SREV}$ that behaves nicely and is easy to compute. Note that $\operatorname{SREV}_{\vec{q}}^{*}$ is essentially computing the revenue of the best item pricing that sells item $i$ with probability at most $q_{i}$, but only counting revenue from cases where the other values are too low to have possibly sold (and actually it undercounts this quantity).

Remark 2.4. In our definitions of $\operatorname{REv}_{q}(D)$ and $\operatorname{SREv}_{\vec{q}}^{*}(D)$, we assume without loss of generality that for every single-dimensional $D$ and $q \in[0,1]$ it is possible to set a price that sells with probability exactly $q$. When $D$ is a continuous distribution, this is true by the intermediate value theorem. When $D$ has a point mass, this is no longer true per se. Fortunately, there are standard methods for reducing the study of arbitrary distributions to continuous ones with arbitrarily small loss. We briefly sketch one, a rounding scheme commonly attributed to Nisan (that appears also in Chawla et al. (2007) and Cai and Huang (2013)):

For any $\epsilon>0, D$ can be "smoothed" into a continuous distribution $D^{\epsilon}$ by multiplying samples from $D$ by a random factor drawn uniformly from $[1,1+\epsilon]$. For any smoothed $D^{\epsilon}$, the desired prices exist by the intermediate value theorem. Using techniques similar in spirit to those of Section 3.1, it is easy (both computationally and conceptually) to convert mechanisms for $D^{\epsilon}$ to mechanisms for $D$, and vice versa, with negligible (dependent on $\epsilon$ ) loss in revenue. Therefore, one may formally study $D^{\epsilon}$ for sufficiently small $\epsilon$, and all results hold with respect to $D$ as well with negligible loss (and taking $\epsilon \rightarrow 0$ results in no loss at all). So, for the remainder of the article, we will assume w.l.o.g. that all distributions are continuous, and therefore the desired prices exist.

We conclude the preliminaries by stating a lemma of Hart and Nisan that we will use. We include the proof below for completeness, as well as to verify that it continues to hold when the valuations are not additive.

Lemma 2.5 (Sub-domain Stitching Special Case (Hart and Nisan 2012)). Rev(D) $\leq$ $\sum_{A} p_{A} \operatorname{Rev}\left(D_{A}\right)$. 
Proof. Let $M$ be an optimal mechanism for selling items with valuations sampled from $D$, and let $\operatorname{Rev}_{M}(D)=\operatorname{Rev}(D)$ denote its revenue. Then, $\operatorname{REv}_{M}(D)=\sum p_{A} \operatorname{Rev}_{M}\left(D_{A}\right)$. Also, for each $A \subseteq$ $[n], \operatorname{Rev}_{M}\left(D_{A}\right) \leq \operatorname{Rev}\left(D_{A}\right)$.

\section{MAIN RESULT: CONSTANT-FACTOR APPROXIMATION FOR SUBADDITIVE BUYER}

THEOREM 3.1. When $D$ is subadditive over independent items, there exists a probability vector $\vec{q}$ such that

$$
\operatorname{Rev}(D) \leq 314 \operatorname{SREV}_{\vec{q}}^{*}(D)+24 \operatorname{BREv}(D) .
$$

Furthermore, $\vec{q}$ can be computed efficiently, as well as an induced item pricing that yields expected revenue at least $\operatorname{SREv}_{\vec{q}}^{*}(D)$.

Proof Outline. We follow the core-tail decomposition framework. First, we provide an approximate core decomposition lemma in Section 3.1. Then, we provide a bound on the contribution of the core with respect to $\max \left\{\mathrm{BREV}, \mathrm{SREV}^{*}\right\}$ in Section 3.2, and a bound on the contribution of the tail with respect to SREV* as a function of the cutoffs chosen in Section 3.3.

For ease of exposition, we simply set $t$ so that the probability of having an empty tail is exactly half; i.e., $p_{\emptyset}=\prod\left(1-p_{i}\right)=1 / 2$. We also set $\vec{q}=\vec{p}$.

\subsection{Approximate Core Decomposition}

In this section, we prove our approximate core decomposition lemma. The key ingredient will be an approximate version of the Marginal Mechanism lemma from Cai and Huang (2013) and Hart and Nisan (2012) for subadditive buyers, stated below:

Lemma 3.2 (Approximate Marginal Mechanism). Let $S, X$ be a partition of $[n]$, and let $D=$ $\left(D^{S}, D^{X}\right)$ be the joint distribution for the valuations of items in $S, X$, respectively, for buyers with subadditive valuations. Then for any $0<\epsilon<1$,

$$
\operatorname{Rev}(D) \leq\left(\frac{1}{\epsilon}+\frac{1}{1-\epsilon}\right) \operatorname{VAL}\left(D^{S}\right)+\frac{1}{1-\epsilon} \mathrm{E}_{v^{S} \leftarrow D^{S}}\left[\operatorname{Rev}\left(D^{X} \mid v^{S}\right)\right]
$$

When $D^{S}$ and $D^{X}$ are independent, this simplifies to

$$
\operatorname{REv}(D) \leq\left(\frac{1}{\epsilon}+\frac{1}{1-\epsilon}\right) \operatorname{VAL}\left(D^{S}\right)+\frac{1}{1-\epsilon} \operatorname{REV}\left(D^{X}\right) .
$$

We outline the proof of Lemma 3.2 below. We first recall the original Marginal Mechanism lemma (that holds for an additive buyer without any multipliers). We provide a complete proof so that the reader can see where the argument fails for subadditive buyers.

Lemma 3.3 (Marginal Mechanism (Cai And Huang 2013; Hart and Nisan 2012)). Let $S \sqcup X$ be any partition of $[n]$, and let $D^{+}$be any distribution over valuation functions such that $v^{+}(U)=$ $v^{+}(U \cap S)+v^{+}(U \cap X)$ for all $U \subseteq n$, and $v^{+}$in the support of $D^{+}$. Let also $D^{S}$ denote $D^{+}$restricted to items in $S$ and $D^{X}$ denote $D^{+}$restricted to items in $X$. Then $\operatorname{Rev}\left(D^{+}\right) \leq \operatorname{VAL}\left(D^{S}\right)+$ $\mathrm{E}_{v^{S} \leftarrow D^{S}}\left[\operatorname{REv}\left(D^{X} \mid v^{S}\right)\right]$.

Proof. We design a mechanism $M^{X}$ (the "Marginal Mechanism") to sell items in $X$ to consumers sampled from $D^{X} \mid v^{S}$ based on the optimal mechanism $M$ for selling items in $S \sqcup X$ to consumers sampled from $D^{+}$. Define $A(v)$ to be the (possibly random) allocation of items awarded to type $v$ in $M$, and $p(v)$ to be the price paid. Let $M^{X}$ first sample a value $v^{S} \leftarrow D^{S}$. The buyer is then invited to report any type $v^{X}$, and $M^{X}$ will award him the items in $A\left(v^{S}, v^{X}\right) \cap X$ and charge him price $p\left(v^{S}, v^{X}\right)-v^{S}\left(A\left(v^{S}, v^{X}\right) \cap S\right)$. In other words, the buyer will receive value from exactly the same 
items in $M^{X}$ as he would have received in $M$, except he receives the actual items in $X$, whereas for items in $S$ he is given a monetary rebate instead of his actual value.

We first claim that if $M$ is truthful, then so is $M^{X}$. The utility of a buyer with type $v^{X}$ for reporting $w^{X}$ to $M^{X}$ can be written as: $v^{X}\left(A\left(v^{S}, w^{X}\right) \cap X\right)+v^{S}\left(A\left(v^{S}, w^{X}\right) \cap S\right)-p\left(v^{S}, w^{X}\right)=$ $\left(v^{S}, v^{X}\right)\left(A\left(v^{S}, w^{X}\right)\right)-p\left(v^{S}, w^{X}\right)$, which is exactly the utility of a buyer with type $\left(v^{S}, v^{X}\right)$ for reporting $\left(v^{S}, w^{X}\right)$ to $M$. As $M$ was truthful, we know that a buyer with type $\left(v^{S}, v^{X}\right)$ maximizes utility when reporting $\left(v^{S}, v^{X}\right)$ over all possible $\left(v^{S}, w^{X}\right)$. Therefore, a buyer with type $v^{X}$ prefers to tell the truth as well.

Finally, we just have to compute the revenue of $M^{X}$. For each $v^{S}$, the marginal mechanism provides a concrete mechanism for the distribution $D^{X} \mid v^{S}$ that attains revenue at least $\operatorname{Rev}\left(D^{+} \mid v^{S}\right)-v^{S}(S)$. So $\operatorname{Rev}\left(D^{X} \mid v^{S}\right) \geq \operatorname{Rev}\left(D^{+} \mid v^{S}\right)-v^{S}(S)$. Taking an expectation over all $v^{S}$ (and an application of sub-domain stitching) yields the lemma.

Notice that it is crucial in the proof above that the buyer's value could be written as $v^{S}(\cdot)+v^{X}(\cdot)$. Otherwise the auctioneer does not know how much to "reimburse" the buyer, since the correct amount depends on the buyer's private information. The buyer can then manipulate his own report $v^{X}$ to influence how much he gets reimbursed for the items in $S$.

A natural approach then, given any distribution $D$ over subadditive valuations, is to define a new value distribution $D^{+}$by redefining all $v(\cdot)$ to satisfy $v(U)=v(U \cap S)+v(U \cap X)$ (it is easy to see that all valuations in the support of $D^{+}$are still subadditive). Unfortunately, even though $D^{+}$(first-order stochastically) dominates $D$, due to non-monotonicity we could very well have $\operatorname{Rev}\left(D^{+}\right)<\operatorname{Rev}(D)$. Still, we bound the revenue lost as we move from $D$ to $D^{+}$by making use of tools for turning $\epsilon$-truthful mechanisms into truly truthful ones. Lemma 3.4 and Corollary 3.5 below capture this formally.

Lemma 3.4. Consider two coupled distributions $D$ and $D^{+}$, with $v(\cdot)$ and $v^{+}(\cdot)$ denoting a random sample from each. Define the random function $\delta(\cdot)$ so that $\delta(S)=v^{+}(S)-v(S)$ for all S. Suppose that $\delta(S) \geq 0$ for all $S$ and that $\delta(\cdot)$ also satisfies $\mathrm{E}_{D}\left[\max _{S \subseteq[n]}\{\delta(S)\}\right] \leq \bar{\delta}$. Then, for any $0<\epsilon<1$,

$$
\operatorname{Rev}\left(D^{+}\right) \geq(1-\epsilon)(\operatorname{Rev}(D)-\bar{\delta} / \epsilon)
$$

Proof. Consider a mechanism $M$ that achieves the optimal revenue $\operatorname{Rev}(D)$. Let $\left(\phi_{v}, p_{v}\right)$ denote the lottery purchased by a buyer with type $v$ in $M$, where $\phi_{v}$ is a (possibly randomized) allocation, and $p$ is a price. Consider now the mechanism $M^{+}$that offers the same menu as $M$, but with all prices discounted by a factor of $(1-\epsilon)$. Let $\left(\phi_{v}^{+}, p_{v}^{+}\right)$denote the lottery that a buyer with type $v^{+}$(coupled with $v$ ) chooses to purchase in $M^{+}$(knowing that she would only pay $(1-\epsilon) p_{v}^{+}$ because of the discount). The following inequalities must hold (we will abuse notation and let $\left.v(\psi)=\mathrm{E}_{S \leftarrow \psi}[v(S)]\right):$

$$
\begin{gathered}
v\left(\phi_{v}\right)-p_{v} \geq v\left(\phi_{v}^{+}\right)-p_{v}^{+} . \\
v^{+}\left(\phi_{v}^{+}\right)-(1-\epsilon) p_{v}^{+} \geq v^{+}\left(\phi_{v}\right)-(1-\epsilon) p_{v} .
\end{gathered}
$$

Now, summing Equations (2) and (3) (then making use of the definition of $\delta(\cdot)$ and the fact that it is non-negative), we have

$$
\begin{aligned}
\epsilon p_{v}^{+}+\delta\left(\phi_{v}^{+}\right) & \geq \epsilon p_{v} \\
\Rightarrow p_{v}^{+} & \geq p_{v}-\delta\left(\phi_{v}^{+}\right) / \epsilon
\end{aligned}
$$


We can now bound the expected revenue by taking an expectation over all valuations:

$$
\begin{aligned}
\operatorname{Rev}\left(D^{+}\right) & \geq \mathrm{E}_{v \leftarrow D}\left[(1-\epsilon) p_{v}^{+}\right] \\
& \geq(1-\epsilon) \mathrm{E}_{v \leftarrow D}\left[p_{v}-\delta\left(\phi_{v}^{+}\right) / \epsilon\right] \\
& \geq(1-\epsilon) \operatorname{REv}(D)-(1-\epsilon) \bar{\delta} / \epsilon .
\end{aligned}
$$

Corollary 3.5. For a given partition of $[n], S \sqcup X$, and distribution $D$ over subadditive valuations, define $D_{S}$ to be $D$ restricted to items in $S$, and $D^{+}$to first sample $v \leftarrow D$, and output $v^{+}(\cdot)$ with $v^{+}(U)=v(U \cap S)+v(U \cap X)$. Then, for all $\epsilon \in(0,1), \operatorname{Rev}(D) \leq \frac{\operatorname{Rev}\left(D^{+}\right)}{1-\epsilon}+\frac{\operatorname{VaL}\left(D_{S}\right)}{\epsilon}$.

Proof. By monotonicity, $v(U) \geq v(U \cap X)$ for all $U, X$. Therefore, $v^{+}(U)-v(U) \leq v(U \cap S) \leq$ $v(S)$ for all $U$. Furthermore, by subadditivity, we have $v^{+}(U) \geq v(U)$ for all $U$. Together, this means that $D$ and $D^{+}$are coupled so that we can set $\delta(U) \leq v(S)$ for all $U$. Therefore, we may set $\bar{\delta}=$ $\operatorname{VAL}\left(D_{S}\right)$ in the hypothesis of Lemma 3.4. The corollary follows by rearranging the inequality.

The proof of Lemma 3.2 is now a combination of Corollary 3.5 and Lemma 3.3.

Proof of Lemma 3.2. Chaining Corollary 3.5 together with Lemma 3.3 we get

$$
\begin{aligned}
& \operatorname{Rev}(D) \leq \frac{\operatorname{Rev}\left(D^{+}\right)}{1-\epsilon}+\frac{\operatorname{VAL}\left(D^{S}\right)}{\epsilon} \\
& \leq \frac{\operatorname{VAL}\left(D^{S}\right)+\mathrm{E}_{v^{S} \leftarrow D^{S}}\left[\operatorname{Rev}\left(D^{X} \mid v^{S}\right)\right]}{1-\epsilon}+\frac{\operatorname{VAL}\left(D^{S}\right)}{\epsilon} \\
& =\left(\frac{1}{1-\epsilon}+\frac{1}{\epsilon}\right) \operatorname{VAL}\left(D^{S}\right)+\frac{1}{1-\epsilon} \mathrm{E}_{v^{S} \leftarrow D^{S}}\left[\operatorname{Rev}\left(D^{X} \mid v^{S}\right)\right],
\end{aligned}
$$

as desired.

We can now provide our approximate core decomposition by combining sub domain stitching (Lemma 2.5) and approximate marginal mechanism (Lemma 3.2).

Lemma 3.6 (Approximate Core Decomposition). For any distribution $D$ that is subadditive over independent items, and any $0<\epsilon<1$,

$$
\operatorname{REV}(D) \leq\left(\frac{1}{\epsilon}+\frac{1}{1-\epsilon}\right) \operatorname{VAL}\left(D_{\emptyset}^{C}\right)+\frac{1}{1-\epsilon} \sum_{A \subseteq[n]} p_{A} \operatorname{REV}\left(D_{A}^{T}\right) .
$$

In particular, for $\epsilon=1 / 2$, we have

$$
\operatorname{Rev}(D) \leq 4 \operatorname{VAL}\left(D_{\emptyset}^{C}\right)+2 \sum_{A \subseteq[n]} p_{A} \operatorname{Rev}\left(D_{A}^{T}\right) .
$$

Proof. By the Approximate Marginal Mechanism Lemma (Lemma 3.2),

$$
\operatorname{REv}\left(D_{A}\right) \leq\left(\frac{1}{\epsilon}+\frac{1}{1-\epsilon}\right) \operatorname{VAL}\left(D_{A}^{C}\right)+\frac{1}{1-\epsilon} \operatorname{Rev}\left(D_{A}^{T}\right) .
$$

Also, for any $A \subseteq[n]$,

$$
\operatorname{VAL}\left(D_{A}^{C}\right) \leq \operatorname{VAL}\left(D_{\emptyset}^{C}\right) .
$$

Finally, by sub-domain stitching (Lemma 2.5):

$$
\begin{aligned}
\operatorname{Rev}(D) & \leq \sum_{A \subseteq[n]} p_{A} \operatorname{Rev}\left(D_{A}\right) \\
& \leq \sum_{A \subseteq[n]} p_{A}\left(\left(\frac{1}{\epsilon}+\frac{1}{1-\epsilon}\right) \operatorname{VAL}\left(D_{A}^{C}\right)+\frac{1}{1-\epsilon} \operatorname{ReV}\left(D_{A}^{T}\right)\right) \\
& \leq\left(\frac{1}{\epsilon}+\frac{1}{1-\epsilon}\right) \operatorname{VAL}\left(D_{\emptyset}^{C}\right)+\frac{1}{1-\epsilon} \sum_{A \subseteq[n]} p_{A} \operatorname{ReV}\left(D_{A}^{T}\right) .
\end{aligned}
$$




\subsection{Core}

Here, we show how to bound $\operatorname{VAL}\left(D_{\emptyset}^{C}\right)$ using $\max \left\{\operatorname{SREV}_{\vec{q}}^{*}(D), \operatorname{BREv}(D)\right\}$. We use a concentration result due to Schechtman (2003) that first requires a definition. Intuitively, the definition below says that a distribution is $c$-Lipschitz if changing the "private information" for a single item cannot change the buyer's value for any set by more than $c$. Moreover, adding/removing a single item cannot change the buyer's value for any set by more than $c$.

Definition 3.7. Let $D^{\vec{x}}$ denote a distribution of private information, $V$ denote a valuation function $V(\vec{x}, \cdot)$, and $D$ denote the distribution that samples $\vec{x} \leftarrow D^{\vec{x}}$ and outputs the function $v(\cdot)=V(\vec{x}, \cdot)$. Then $D$ is c-Lipschitz if for all $\vec{x}, \vec{y}$, and sets $S$ and $T$ we have

$$
|V(\vec{x}, S)-V(\vec{y}, T)| \leq c \cdot\left(|S \cup T|-|S \cap T|+\left|\left\{i \in S \cap T: x_{i} \neq y_{i}\right\}\right|\right) .
$$

Before applying Schechtman's theorem, we show that $D_{\emptyset}^{C}$ is $t$-Lipschitz (recall that $t$ is the cutoff between core and tail).

Lemma 3.8. Let $D$ be any distribution that is subadditive over independent items where each $v(\{i\}) \in[0, c]$ with probability 1 . Then D is c-Lipschitz.

Proof. For any $\vec{x}, \vec{y}, S, T$, let $U=\left\{i \in S \cap T \mid x_{i}=y_{i}\right\}$. Because of no externalities, we must have $V(\vec{x}, U)=V(\vec{y}, U)$, which we will denote by $B$. By monotonicity, we must have $V(\vec{x}, S)$, $V(\vec{y}, T) \geq B$.

Now, by subadditivity and the fact that each $V(\vec{x},\{i\}) \leq c$, we have $V(\vec{x}, S) \leq c(|S|-|U|)+B$. Similarly, we have $V(\vec{y}, T) \leq c(|T|-|U|)+B$.

It's also clear that $|S|-|U| \leq|S \cup T|-|S \cap T|+\left|\left\{i \in S \cap T: x_{i} \neq y_{i}\right\}\right|$ (the RHS is just rewriting the size of $S \cup T-U)$, and that $|T|-|U| \leq|S \cup T|-|S \cap T|+\left|\left\{i \in S \cap T: x_{i} \neq y_{i}\right\}\right|$.

So by everything above, we must have $V(\vec{x}, S), V(\vec{y}, T) \leq B+c(|S \cup T|-|S \cap T|+\mid\{i \in S \cap T$ : $\left.\left.x_{i} \neq y_{i}\right\} \mid\right)$. Therefore, $V(\vec{x}, S), V(\vec{y}, S) \in\left[B, B+c\left(|S \cup T|-|S \cap T|+\left|\left\{i \in S \cap T: x_{i} \neq y_{i}\right\}\right|\right)\right]$, completing the proof.

Corollary 3.9. $D_{\emptyset}^{C}$ is t-Lipschitz.

Now we state Schechtman's theorem and apply it to bound $\operatorname{VAL}\left(D_{\emptyset}^{C}\right)$.

Theorem 3.10 (Schechtman 2003). Suppose that D is a distribution that is subadditive over independent items and c-Lipschitz. Then, for any parameters $q, a, k>0$,

$$
\operatorname{Pr}_{v \leftarrow D}[v([n]) \geq(q+1) a+k \cdot c] \leq \operatorname{Pr}[v([n]) \leq a]^{-q} q^{-k} .
$$

In particular, if $a$ is the median of $\left.v([n])\right|_{v \leftarrow D}$ and $q=2$, we have

$$
\operatorname{Pr}_{v \leftarrow D}[v([n]) \geq 3 a+k \cdot c] \leq 4 \cdot 2^{-k} .
$$

Corollary 3.11. Suppose that $D$ is a distribution that is subadditive over independent items and $c$-Lipschitz. If a is the median of $\left.v([n])\right|_{v \leftarrow D}$, then $\mathrm{E}_{v \leftarrow D}[v([n])] \leq 3 a+4 c / \ln 2$.

Proof. $\mathrm{E}[v([n])]=\int_{0}^{\infty} \operatorname{Pr}[v([n])>y] d y$. We can upper bound this using the minimum of 1 and the bound provided in Theorem 3.10 to yield

$$
\mathrm{E}[v([n])] \leq 3 a+\int_{0}^{\infty} 4 \cdot 2^{-y / c} d y=3 a+4 c / \ln 2 .
$$

Proposition 3.12. $\operatorname{VAL}\left(D_{\emptyset}^{C}\right) \leq 6 \mathrm{BReV}+4 t / \ln 2$. 
Proof. Since $a$ is the median of $v([n])$, we can set price $a$ on the grand bundle and extract revenue at least $a / 2$. Therefore, $\operatorname{BREv} \geq a / 2$. By Corollaries 3.11 and 3.9, we have $\operatorname{VAL}\left(D_{\emptyset}^{C}\right) \leq 3 a+$ $4 t / \ln (2)$, so $\operatorname{VAL}\left(D_{\emptyset}^{C}\right) \leq 6 \mathrm{BREV}+4 t / \ln (2)$.

Finally, if the cutoff $t$ is not too large, we can recover a constant fraction of it by selling each item separately.

LEMmA 3.13. $\operatorname{SREV}_{\vec{p}}^{*} \geq t \cdot p_{\emptyset}\left(1-p_{\emptyset}\right)$. In particular, if we choose $t$ so that $p_{\emptyset}=1 / 2$, then $\mathrm{SREV}_{\vec{p}}^{*} \geq$ $t / 4$.

Proof. Clearly $\operatorname{Rev}_{p_{i}}\left(D_{i}\right) \geq p_{i} t$, as we could set a price of $t$ for item $i$. So $\operatorname{SREV}_{\vec{p}}^{*}=$ $p_{\emptyset} \sum_{i} \operatorname{Rev}_{p_{i}}\left(D_{i}\right) \geq p_{\emptyset} t \sum_{i} p_{i}$ Finally, we observe that $\sum_{i} p_{i}$ is exactly the expected number of items in the tail, and $p_{\emptyset}$ is the probability that zero items are in the tail. So we clearly have $\sum_{i} p_{i} \geq 1-p_{\emptyset}$.

Combining Proposition 3.12 and Lemma 3.13 then yields:

Proposition 3.14

$$
\operatorname{VAL}\left(D_{\emptyset}^{C}\right) \leq 6 \mathrm{BREV}+24 \operatorname{SREV}_{\vec{p}}^{*} .
$$

Proof. By Proposition 3.12, $\operatorname{VAL}\left(D_{\emptyset}^{C}\right) \leq 6 \mathrm{BRev}+4 t / \ln (2)$. By Lemma 3.13, $t \leq 4 \mathrm{SReV}_{\vec{p}}^{*}$. As $16 / \ln (2) \leq 24$, we get the proposition as desired.

\subsection{Tail}

We now show that the revenue from the tail can be approximated by $\operatorname{SRV}_{\vec{q}}^{*}$. We begin by proving a much weaker bound on the optimum revenue for any distribution of subadditive valuations over independent items:

LEMMA 3.15.

$$
\operatorname{REV}(D) \leq 6 n^{\log _{2} 6} \sum_{i} \operatorname{REV}\left(D_{i}\right)
$$

Proof. Babaioff et al. (2014) prove that $\operatorname{Rev} \leq n \sum_{i} \operatorname{Rev}\left(D_{i}\right)$ for an additive buyer by recursively reducing the number of items by one at each step. Unfortunately, each step of the induction uses the Marginal Mechanism Lemma; when applying the approximate variant for subadditive valuations, we would incur an exponential factor.

Instead, we use a slightly more complicated argument along the lines of Hart and Nisan (2012) that halves the number of items in each step. Let $S$ and $X$ be a partition of $[n]$ into subsets of size at most $\lceil n / 2\rceil$. Let $D_{S \geq X}$ be the distribution over valuations, which is the same as $D$ whenever $v(S) \geq v(T)$, and has valuation zero otherwise. Similarly, let $D_{S<X}$ be the distribution that is equal to $D$ on $v(S)<(T)$. Then, by sub-domain stitching (Lemma 2.5), we have

$$
\operatorname{Rev}(D) \leq \operatorname{Rev}\left(D_{S \geq X}\right)+\operatorname{Rev}\left(D_{S<X}\right) .
$$

Now, by the Approximate Marginal Mechanism Lemma,

$$
\operatorname{REV}\left(D_{S \geq X}\right) \leq\left(\frac{1}{\epsilon}+\frac{1}{1-\epsilon}\right) \operatorname{VAL}\left(D_{S \geq X}^{X}\right)+\frac{1}{1-\epsilon} \mathrm{E}_{v^{X} \leftarrow D_{S \geq X}^{X}}\left[\operatorname{REv}\left(D_{S \geq X}^{S} \mid v^{X}\right)\right] .
$$

One mechanism for selling items in $S$ is to sample $v^{X} \leftarrow D_{S \geq X}^{X}$, and then use a mechanism that achieves $\operatorname{Rev}\left(D_{S \geq X}^{S} \mid v^{X}\right)$. Thus we have

$$
\operatorname{REv}\left(D^{S}\right) \geq \mathrm{E}_{v^{X} \leftarrow D_{S \geq X}^{X}}\left[\operatorname{Rev}\left(D_{S \geq X}^{S} \mid v^{X}\right)\right]
$$


Another way to sell items in $S$ is to again sample $v^{X} \leftarrow D_{S \geq X}^{X}$, and offer the entire bundle for price $v^{X}(X)$. Therefore, we also have

$$
\operatorname{Rev}\left(D^{S}\right) \geq \mathrm{E}_{v \leftarrow D}[v(X) \mid(v(S) \geq v(X))]=\mathrm{E}_{v \leftarrow D_{S \geq X}}[v(X)]=\operatorname{VAL}\left(D_{S \geq X}^{X}\right)
$$

Combining Equations (5)-(7), we have

$$
\operatorname{REv}\left(D_{S \geq X}\right) \leq\left(\frac{1}{\epsilon}+\frac{2}{1-\epsilon}\right) \operatorname{Rev}\left(D^{S}\right) .
$$

By symmetry, the same holds for $\operatorname{Rev}\left(D_{S<X}\right)$ and $\operatorname{Rev}\left(D^{X}\right)$. Therefore, using Equation (4),

$$
\operatorname{Rev}(D) \leq\left(\frac{1}{\epsilon}+\frac{2}{1-\epsilon}\right)\left(\operatorname{Rev}\left(D^{S}\right)+\operatorname{Rev}\left(D^{X}\right)\right) .
$$

Applying the recursion $\lceil\log n\rceil$ times, we have

$$
\operatorname{ReV}(D) \leq\left(\frac{1}{\epsilon}+\frac{2}{1-\epsilon}\right)^{\log _{2} n+1} \sum_{i} \operatorname{Rev}\left(D_{i}\right)=\left(\frac{1}{\epsilon}+\frac{2}{1-\epsilon}\right) n^{\log _{2}\left(\frac{1}{\epsilon}+\frac{2}{1-\epsilon}\right)} \sum_{i} \operatorname{Rev}\left(D_{i}\right) .
$$

Choosing $\epsilon=1 / 2$ yields $\left(\frac{1}{\epsilon}+\frac{2}{1-\epsilon}\right)=6$.

Note that in Lemma 3.15, $\sum_{i} \operatorname{Rev}\left(D_{i}\right)$ is not the same as $\operatorname{SREv}(D)$ as the buyer is not necessarily additive. In fact, they can be off by a factor of $n$ (in the case of a unit-demand buyer). Nonetheless, this weak bound suffices for our analysis of the tail, which is summarized in Proposition 3.16 below. Essentially, the proposition amplifies the bound in Lemma 3.15 greatly by making use of the fact that it is unlikely to see multiple items in the tail.

Proposition 3.16. Recall that $p_{i}=\operatorname{Pr}[v(\{i\})>t]$, and $p_{\emptyset}=\prod_{i}\left(1-p_{i}\right)$. Then

$$
\sum_{A} p_{A} \operatorname{REv}\left(D_{A}^{T}\right) \leq \frac{6}{p_{\emptyset}}\left(1+7 \ln \left(1 / p_{\emptyset}\right)+6 \ln \left(1 / p_{\emptyset}\right)^{2}+\ln \left(1 / p_{\emptyset}\right)^{3}\right) \cdot \operatorname{SREv}_{\vec{p}}^{*}
$$

In particular, if we choose $t$ so that $p_{\emptyset}=1 / 2$, then $\sum_{A} p_{A} \operatorname{REV}\left(D_{A}^{T}\right) \leq 109 \cdot \operatorname{SREV}_{\vec{p}}^{*}$.

Proof. Our proof builds on the intuition that the number of items in the tail is typically very small. By Lemma 3.15, we have that

$$
\begin{aligned}
\sum_{A \subseteq[n]} p_{A} \operatorname{Rev}\left(D_{A}^{T}\right) & \leq \sum_{A \subseteq[n]} p_{A} 6|A|^{\log _{2} 6} \sum_{i \in A} \operatorname{Rev}\left(D_{i}^{T}\right) \\
& =6 \sum_{i \in[n]} p_{i} \operatorname{Rev}\left(D_{i}^{T}\right) \sum_{A \ni i}|A|^{\log _{2} 6} p_{A} / p_{i}
\end{aligned}
$$

For any $i$, the expression $\sum_{A \ni i}|A| p_{A} / p_{i}$ is also the expected number of items in the tail, conditioning on $i$ being in the tail. Similarly, $\sum_{A \ni i}|A|^{\log _{2} 6} p_{A} / p_{i}$ is the expected value of (\# items) log $^{\log ^{6}}$. Let $b_{j}$ be the indicator random variable that is 1 whenever item $j$ is in the tail. Noting that $\log _{2} 6<3$ 
and each $b_{j}$ is 1 with probability exactly $p_{j}$ and the $b_{j}$ 's are independent, we have

$$
\begin{aligned}
\sum_{A \ni i}|A|^{\log _{2} 6} p_{A} / p_{i} \leq & \mathrm{E}_{b_{j}}\left[\left(1+\sum_{j \neq i} b_{j}\right)^{3}\right] \\
= & \mathrm{E}_{b_{j}}\left[1+3\left(\sum_{j \neq i} b_{j}\right)+3\left(\sum_{j \neq i} b_{j}\right)^{2}+\left(\sum_{j \neq i} b_{j}\right)^{3}\right] \\
= & 1+3 \mathrm{E}\left[\sum_{j \neq i} b_{j}\right]+3 \mathrm{E}\left[\sum_{j \neq i} b_{j}^{2}+\sum_{k \neq j \neq i} b_{j} b_{k}\right] \\
& +\mathrm{E}\left[\sum_{j \neq i} b_{j}^{3}+3 \sum_{k \neq j \neq i} b_{j}^{2} b_{k}+\sum_{l \neq k \neq j \neq i} b_{j} b_{k} b_{l}\right] \\
= & \left.1+7 \mathrm{E}\left[\sum_{j \neq i} b_{j}\right]+6 \mathrm{E}\left[\sum_{k \neq j \neq i} b_{j} b_{k}\right]+\mathrm{E} \sum_{l \neq k \neq j \neq i} b_{j} b_{k} b_{l}\right] \\
\leq & 1+7 \sum_{j \neq i} p_{j}+6\left(\sum_{j \neq i} p_{j}\right)^{2}\left(\sum_{j \neq i} p_{j}\right)^{3} .
\end{aligned}
$$

Equation (9) follows because $b_{j} \in\{0,1\}$. We continue to bound the last line as a function of just $p_{\emptyset}$. Note that $e^{-\sum_{i} p_{i}} \geq \prod_{i}\left(1-p_{i}\right)=p_{\emptyset}$, and therefore we have $\sum_{i} p_{i} \leq \ln \left(1 / p_{\emptyset}\right)$. Combining with Equations (8) and (10), we have

$$
\sum_{A \subseteq[n]} p_{A} \operatorname{REV}\left(D_{A}^{T}\right) \leq 6\left(1+7 \ln \left(1 / p_{\emptyset}\right)+6 \ln \left(1 / p_{\emptyset}\right)^{2}+\ln \left(1 / p_{\emptyset}\right)^{3}\right) \cdot \sum_{i \in[n]} p_{i} \operatorname{REV}\left(D_{i}^{T}\right) .
$$

Now, we have to interpret $p_{i} \operatorname{Rev}\left(D_{i}^{T}\right)$. We claim that in fact this is exactly $\operatorname{Rev}_{p_{i}}\left(D_{i}\right)$. Why? It's clear that the optimal reserve for $D_{i}^{T}$ is at least $t$, as $D_{i}^{T}$ is not supported below $t$. It's also easy to see that for any reserve $r_{i} \geq t$, that the revenue obtained by selling to $D_{i}^{T}$ is exactly $r_{i}$. $\operatorname{Pr}\left[v(\{i\})>r_{i}\right] / p_{i}$, and therefore the same $r_{i} \geq t$ that is optimal for $D_{i}$ is also optimal for $D_{i}^{T}$, and $p_{i} \operatorname{Rev}\left(D_{i}^{T}\right)=\operatorname{Rev}_{p_{i}}\left(D_{i}\right)$. Therefore,

$$
\sum_{A \subseteq[n]} p_{A} \operatorname{Rev}\left(D_{A}^{T}\right) \leq 6\left(1+7 \ln \left(1 / p_{\emptyset}\right)+6 \ln \left(1 / p_{\emptyset}\right)^{2}+\ln \left(1 / p_{\emptyset}\right)^{3}\right) \cdot \sum_{i \in[n]} \operatorname{Rev}_{p_{i}}\left(D_{i}\right) .
$$

Plug in $\operatorname{SREV}_{\vec{p}}^{*}=p_{\emptyset} \sum_{i \in[n]} \operatorname{REV}_{p_{i}}\left(D_{i}\right)$ to complete the proof.

Note that Theorem 3.1 is now a corollary of Proposition 3.16, Proposition 3.14, and Lemma 3.6 (setting $\epsilon=1 / 2$ ). That the desired $\vec{q}$ can be computed efficiently is easy to see: simply do a binary search over cutoffs $t$ until we find one that induces $p_{\emptyset}=1 / 2$. It is also easy to find an item pricing that guarantees revenue at least $\operatorname{SREV}_{\vec{q}}^{*}$ : for each item $i$, simply find the optimal reserve for $D_{i}$, subject to that reserve being at least $t$. Finally, notice that the only bundle price we ever need to set to obtain our guarantee is the median of $v([n])$, when $v(\cdot) \leftarrow D_{\emptyset}^{C}$. It is also easy to see that our bounds degrade smoothly if we set a price that only approximates the median instead. For a discussion of exactly what access to $D$ suffices in order for these prices/cutoffs to be truly easy to find, we refer the reader to Babaioff et al. (2014). We note here just that it should be clear that any reasonable, even minimal, access to $D$ does indeed suffice. 


\section{SIMPLE AUCTIONS AND APPROXIMATE REVENUE MONOTONICITY}

In this section, we explore the rich connection between approximately optimal simple auctions, and approximate revenue monotonicity. By approximate revenue monotonicity, we formally mean the following:

Definition 4.1. We say that a class of distributions is $\alpha$-approximately revenue monotone if for any two distributions $D$ and $D^{+}$in that class such that $D^{+}$first-order stochastically dominates $D$ (recall that we $D^{+}$first-order stochastically dominates $D$ if they can be coupled so that when we sample $v^{+}$from $D^{+}$and $v$ from $D$ we have $v^{+}(S) \geq v(S)$ for all $\left.S\right), \alpha \cdot \operatorname{Rev}\left(D^{+}\right) \geq \operatorname{Rev}(D)$.

In the rest of the section, we observe that subadditive valuations over independent items are $\alpha$-approximately monotone for some constant factor (Section 4.1). We also note that a (significantly) tighter approximate monotonicity would yield a better factor of approximation in Theorem 3.1 (Section 4.2). Finally, for the class of (possibly correlated) additive valuations over $n$ items, we prove a reduction from approximate revenue monotonicity to approximately optimal simple auctions (that loses a factor of $n$ ). Then we use an infinite gap between max $\{$ BREv, SREv $\}$ and REV for two correlated items due to Hart and Nisan (2013) to prove an infinite lower bound on approximate revenue monotonicity (Section 4.3).

\subsection{Approximately Optimal Simple Auctions Imply Approximate Monotonicity}

As a corollary of our main theorem (Theorem 3.1) we deduce constant-factor approximate monotonicity for subadditive valuations over independent items:

COROLlaRy 4.2. The class of subadditive valuations over independent items is 338-approximately monotone.

Similarly from the 6-approximation of Babaioff et al. for additive yields.

COROLlary 4.3. The class of additive valuations over independent items is 6-approximately monotone.

Proof. For additive functions, BREv and SREv constitute separate Myerson's auctions, and are therefore revenue monotone. Thus,

$$
\begin{aligned}
6 \operatorname{Rev}\left(D^{+}\right) & \geq 6 \max \left\{\operatorname{BRev}\left(D^{+}\right), \operatorname{SRev}\left(D^{+}\right)\right\} \\
& \geq 6 \max \{\operatorname{BRev}(D), \operatorname{SRev}(D)\} \geq \operatorname{Rev}(D) .
\end{aligned}
$$

For subadditive functions, $\operatorname{SREv}(D)$ is no longer monotone, but $\operatorname{SREv}_{\vec{q}}^{*}(D)$ is. This is because $\operatorname{SREv}_{q}\left(D_{i}\right)$ is clearly monotone, and $\operatorname{SREV}_{\vec{q}}^{*}$ is just a (scaled) sum of $\operatorname{SREv}_{q_{i}}\left(D_{i}\right)$. So we get that there exists a $\vec{q}$ such that

$$
\begin{aligned}
338 \operatorname{Rev}\left(D^{+}\right) & \geq 338 \max \left\{\operatorname{BRev}\left(D^{+}\right), \operatorname{SRev}_{\vec{q}}^{*}\left(D^{+}\right)\right\} \\
& \geq 338 \max \left\{\operatorname{BRev}(D), \operatorname{SREv}_{\vec{q}}^{*}(D)\right\} \geq \operatorname{Rev}(D) .
\end{aligned}
$$

\subsection{Approximate Monotonicity Implies Approximately Optimal Simple Auctions}

A closer look at the proof of our main theorem also yields the converse of the above corollaries, namely: a tighter approximate monotonicity for subadditive valuations would yield an improved factor of approximation by simple auctions, as well as a simpler proof.

COROLlary 4.4. If the class of subadditive valuations over independent items is $\alpha$-approximately monotone, then

$$
\operatorname{REV} \leq \alpha((37 \alpha+24) \operatorname{SREV}+6 \mathrm{BREV})
$$


Proof (Sкетсн). Recall that in the proof of the Approximate Marginal Mechanism lemma (Lemma 3.2), we made use of Lemma 3.4 to bound the gap between $\operatorname{Rev}\left(D^{+}\right)$and $\operatorname{Rev}(D)$, where $D^{+}$ first-order stochastically dominated $D$. Instead of the $\epsilon$-truthful to truthful reduction, we could derive $\alpha \operatorname{Rev}\left(D^{+}\right) \geq \operatorname{ReV}(D)$ from approximate monotonicity. Then, we can directly apply Lemma 3.3 to get

instead of

$$
\operatorname{REv}(D) \leq \alpha\left(\operatorname{VAL}\left(D^{S}\right)+\mathrm{E}_{v^{S} \sim D^{S}}\left[\operatorname{Rev}\left(D^{X} \mid \mathbf{v}^{S}\right)\right]\right)
$$

$$
\operatorname{REv}(D) \leq 4 \operatorname{VAL}\left(D^{S}\right)+2 \mathrm{E}_{v^{S} \sim D^{S}}\left[\operatorname{REv}\left(D^{X} \mid \mathbf{v}^{S}\right)\right] .
$$

If $\alpha \leq 2$, this indeed yields a tighter approximation.

\subsection{Correlated Distributions Are Not Approximately Monotone}

So far we've shown that (for some valuation classes) approximately optimal simple mechanisms imply approximate revenue-monotonicity. Are all classes approximately revenue-monotone? In this section, we provide a reduction from an instance where max $\{$ SREv, BREv $\}$ does not approximate REv to show an infinite non-monotonicity for correlated items. We first prove a reduction from gaps between BREv and REv to non-monotonicity.

Proposition 4.5. Let $D$ be a distribution over subadditive valuations for $n$ items for which $\operatorname{Rev}(D)>c \cdot \operatorname{BREv}(D)$. Then any class of distributions containing $D$ and all single-dimensional distributions ${ }^{5}$ is not $(c / n)$-approximately revenue monotone.

Proof. We define $D^{+}$as follows. First, sample $v \leftarrow D$. Then let $i^{*}=\arg \max _{i}\{v(\{i\})\}$. Now, set $v^{+}(S)=v\left(\left\{i^{*}\right\}\right) \cdot|S|$ for all $|S|$. By subadditivity, it's clear that $D^{+}$first order stochastically dominates $D$. Now, however, $D^{+}$is a single-dimensional distribution, meaning that $\operatorname{BREv}\left(D^{+}\right)=$ $\operatorname{Rev}\left(D^{+}\right)$(Myerson 1981; Riley and Zeckhauser 1983). Finally, we just need to compare $\operatorname{BREv}(D)$ to $\operatorname{BREv}\left(D^{+}\right)$.

Note that by monotonicity, we have $v^{+}([n]) \leq n \cdot v([n])$ for all $v, v^{+}$. Therefore, for any price $p$, if $v^{+}([n])>p, v([n])>p / n$. This immediately implies that $\operatorname{BRev}(D) \geq \operatorname{BRev}\left(D^{+}\right) / n$ : let $p$ be the optimal reserve for the grand bundle under $D^{+}$, then setting price $p / n$ sells with at least the same probability under $D$. Putting both observations together, we see that: $\operatorname{Rev}(D)>c \operatorname{BRev}(D) \geq$ $(c / n) \operatorname{BRev}\left(D^{+}\right)=(c / n) \operatorname{Rev}\left(D^{+}\right)$, meaning that any class containing $D$ and $D^{+}$is not $(c / n)-$ approximately monotone.

We apply Proposition 4.5 to a theorem of Hart and Nisan.

THEOREM 4.6 (HART AND NISAN 2013). There exists a distribution D over correlated additive valuations for two items such that $\operatorname{BREv}(D) \leq 1 / 2$, and $\operatorname{Rev}(D)=\infty$.

COROllary 4.7. There exist distributions $D$ and $D^{+}$over correlated additive valuations for two items such that $D^{+}$first-order stochastically dominates $D, \operatorname{REv}\left(D^{+}\right)=1$, and yet $\operatorname{Rev}(D)=\infty$. Therefore, the class of correlated additive valuations for two items is not c-approximately revenue monotone for any finite $c$.

\section{APPROXIMATE MARGINAL MECHANISM AND CORE DECOMPOSITION FOR MANY BIDDERS}

In this section, we provide a complete proof of the Marginal Mechanism and Core Decomposition lemmas that apply to many bidders. The statements are essentially the same, but require more extensive notation, which we develop in the next section.

\footnotetext{
${ }^{5} \mathrm{~A}$ distribution is single-dimensional if for all $v$ in its support, $v(S)=c|S|$ for some value $c$.
} 
The main technical challenge is extending Lemma 3.4 to many buyers; recall that this is the lemma that lower bounds the revenue from a distribution $D^{+}$of additive-across-a-partition valuations, in terms of the revenue from the original distribution $D$ and the expected difference $\bar{\delta}$ between $D$ and $D^{+}$, i.e., given a black-box mechanism for $D$, we would like to create a new mechanism for $D^{+}$. How can we preserve incentive compatibility when the buyers have different incentives? In Section 3.1 we do this for a single buyer by giving the buyer the outcome (allocation and price), of the possible outcomes for all types in the original mechanism, which is optimal for her new valuation. Incentive compatibility is now guaranteed, and we simply need to bound the revenue.

When multiple buyers are involved, there is a problem with this approach: incentive compatibility of any buyer depends also on the distribution of types reported by other buyers; thus we cannot simply let any buyer report any type she wants. To overcome this challenge, we use a technique due to Bei and Huang (2011), Hartline et al. (2011), and Daskalakis and Weinberg (2012), which guarantees that each buyer observes the correct distribution of types on the other buyers. For buyer $i$, we sample an additional $r-1$ replica types from $D_{i}^{+}$, and $r$ surrogate types from $D_{i}$. Given the real buyer's type, we create (in an incentive compatible manner) a complete matching between replicas and surrogates. Since the real buyer's type is sampled from the same distribution as the replicas, she is equally likely to be matched to any of the surrogates. Thus the distribution over the surrogate type that is matched to the buyer $i$ is exactly the original distribution $D_{i}$; i.e., all other buyers observe the "correct" distribution. The mechanism is now Bayesian incentive compatible, and further analysis shows that the lower bound on the revenue is preserved.

\subsection{Notation and Statement}

There are $m$ bidders and $n$ items. We now say that an item $i$ is in the tail if there is any buyer who values item $i$ above the core-tail cutoff, and item $i$ is in the core if every buyer values it below the cutoff. $D$ is now the joint distribution of all buyers valuation functions. We study Bayesian Incentive Compatible (BIC) mechanisms. A mechanism is BIC if it is in every buyer's best interest to report truthfully, conditioned on other buyers reporting truthfully as well. Formally, let $\phi_{j}(v)$ denotes the (random) allocation awarded to bidder $j$ when reporting type $v$; we slightly abuse notation by letting $v(\phi)$ denote the expected utility, over any randomness in the mechanism and the other bidders sampling their types, that a bidder with type $v$ gains from a random allocation $\phi$; let $p_{j}(v)$ denote the expected price paid by bidder $j$ when reporting type $v$ over the same randomness. Finally, a mechanism is BIC iff for all $j, v, v^{\prime}, v\left(\phi_{j}(v)\right)-p_{j}(v) \geq v\left(\phi_{j}\left(v^{\prime}\right)\right)-p_{j}\left(v^{\prime}\right)$. We use the following notation.

$-D_{j}$ : The distribution of $v_{j}(\cdot)$, the valuation function for bidder $j$. We assume that $D$ is a product distribution. That is, $D=\times_{j} D_{j}$.

$-D_{A}$ : the distribution $D$, conditioned on $A$ being exactly the set of items in the tail.

$-D_{A}^{T}$ : the distribution $D_{A}$ restricted just to items in the tail (i.e., $A$ ).

$-D_{A}^{C}$ : the distribution $D_{A}$ restricted just to items in the core (i.e., $\bar{A}$ ).

$-p_{i}$ : the probability that element $i$ is in the tail.

$-p_{A}$ : the probability that $A$ is exactly the set of items in the tail (note that $p_{\{i\}} \neq p_{i}$ ).

$-\operatorname{Rev}(D)$ : The maximum revenue obtainable via a BIC mechanism from buyers with valuation profile drawn from $D$.

$-\operatorname{VAL}(D)=\mathrm{E}_{v \leftarrow D}\left[\max _{S_{1} \sqcup \ldots \sqcup S_{m}}\left\{\sum_{j} v_{j}\left(S_{j}\right)\right\}\right]$ : the expected welfare of the VCG mechanism when buyers are drawn from $D$.

$-\operatorname{REV}^{M}(D)$ : The revenue of a BIC mechanism $M$ when buyers drawn from $D$ play truthfully. 
We are finally ready to state the approximate core decomposition lemma for many buyers:

LEMma 5.1. For any distribution $D=\times_{j} D_{j}$ with each $D_{j}$ subadditive over independent items, and any $0<\epsilon<1$,

$$
\operatorname{Rev}(D) \leq\left(\frac{1}{\epsilon}+\frac{1}{1-\epsilon}\right) \operatorname{VAL}\left(D_{\emptyset}^{C}\right)+\frac{1}{1-\epsilon} \sum_{S \subseteq[n]} p_{A} \operatorname{ReV}\left(D_{A}^{T}\right) .
$$

Proof. Follows from Theorem 5.2 below, together with the arguments used in Section 3.1 and in Babaioff et al. (2014).

\subsection{The Mechanism}

First, we describe the reduction we will use (which is essentially the same as the $\epsilon$-BIC to BIC reduction used in Daskalakis and Weinberg (2012), but without some technical hardships since we aren't concerned with runtime-our proof never actually runs this procedure). Below, $D^{+}$denotes any product distribution that first-order stochastically dominates $D$, and $\delta_{j}(\cdot)$ denotes the random function $v_{j}^{+}(\cdot)-v_{j}(\cdot)$ when couples $v^{+}$and $v$ are sampled jointly from $D^{+}$and $D$. Note that $\delta_{j}(S) \geq$ 0 for all $\delta_{j}, S$. We will also abuse notation and refer to $\delta_{j}$ as the distribution over $\delta_{j}(\cdot)$ as well (so we can write terms like $\operatorname{VAL}(\delta))$.

Phase 1, Surrogate Sale:

(1) Let $M$ be any BIC mechanism for buyers from $D$. Multiply all prices charged by $M$ by $(1-\epsilon)$ and call the new mechanism $M^{\epsilon}$. Interpret the $\epsilon$ fraction of prices given back as rebates.

(2) For each bidder $j$, create $r-1$ replicas sampled i.i.d. from $D_{j}^{+}$and $r$ surrogates sampled i.i.d. from $D_{j}$. Let $r \rightarrow \infty$.

(3) Ask each bidder to report $v_{j}(\cdot)$.

(4) Create a weighted bipartite graph with replicas (and bidder $j$ ) on the left and surrogates on the right. The weight of an edge between a replica (or bidder $j$ ) with type $r_{j}(\cdot)$ and surrogate of type $s_{j}(\cdot)$, is the utility of $r_{j}$ for the expected outcome of $M^{\epsilon}$ when reporting $s_{j}$. That is, the weight of the edge is $r_{j}\left(\phi_{j}^{\epsilon}\left(s_{j}\right)\right)-p_{j}^{\epsilon}\left(s_{j}\right)$.

(5) Compute a maximum matching and VCG prices in this bipartite graph; we henceforth refer to it as the VCG matching. If a replica (or bidder $j$ ) is unmatched in the VCG matching, add an edge to a random unmatched surrogate. (Notice that some replicas may indeed be unmatched if the gain negative utility from the allocation and prices corresponding to some surrogates.) The surrogate selected for bidder $j$ is whomever she is matched to.

\section{Phase 2, Surrogate Competition:}

(1) Let $s_{j}$ denote the surrogate chosen to represent bidder $j$ in phase one, and let $\vec{s}$ denote the entire surrogate profile (i.e., the ones matched to the real buyers). Have the surrogates play $M^{\epsilon}$.

(2) If bidder $j$ was matched to her surrogate through VCG, charge them the VCG price and award them $M_{j}^{\epsilon}(\vec{s})$. Recall that this has an allocation and price component; the price is added onto the VCG price. If bidder $j$ was matched to a random surrogate after VCG, award them nothing and charge them nothing.

THEOREM 5.2. Let $M^{\prime}$ denote the mechanism of the process above, starting from any BIC mechanism $M$ for consumers drawn from $D$. Then $M^{\prime}$ is BIC for consumers drawn from $D^{+}$. Furthermore, for any desired $\epsilon \in(0,1)$, we can have: $\operatorname{ReV}^{M^{\prime}}\left(D^{+}\right) \geq(1-\epsilon) \cdot\left(\operatorname{ReV}^{M}(D)-\operatorname{VAL}(\delta) / \epsilon\right)$. 
In the theorem statement above, note that by $\operatorname{VAL}(\delta)$, we mean the expected welfare of the VCG mechanism when buyers with types distributed according to $\delta=\times_{j} \delta_{j}$ play.

\subsection{Proof Outline}

The reduction is nearly identical to the reduction employed in Daskalakis and Weinberg (2012) (which is itself inspired by Bei and Huang (2011) and Hartline et al. (2011)). We provide complete proofs of all claims for completeness, noting that many of these claims can also be found in Bei and Huang (2011), Hartline et al. (2011), and Daskalakis and Weinberg (2012). We provide appropriate citations by each statement. Below is the proof outline, taken from Daskalakis and Weinberg (2012).

(1) If bidder $j$ plays $M^{\prime}$ truthfully, then the distribution of surrogates matched to bidder $j$ is $D_{j}$. Therefore, the value of each edge is calculated correctly as the expected utility of a replica with type $r$ for being represented by a surrogate of type $s$ in $M^{\epsilon}$.

(2) Because each bidder is participating in a VCG auction for their surrogate, and the value of each edge is calculated correctly, whenever all other bidders tell the truth, it is in bidder $j$ 's interest to tell the truth as well. Therefore, $M^{\prime}$ is BIC.

(3) The revenue made from bidder $j$ is at least the price paid by their surrogate if bidder $j$ is matched in VCG, and 0 otherwise.

(4) There exists a near-perfect matching that matches each replica to a nearly-identical surrogate (modulo $\left.\delta_{j}(\cdot)\right)$. If VCG used this matching, we would have $\operatorname{REV}^{M^{\prime}}(D)=(1-$ $\epsilon) \operatorname{ReV}^{M}(D)$.

(5) The rebates allow us to bound the revenue lost by selecting the VCG matching instead of this near-perfect matching as a function of $\operatorname{VaL}(\delta)$ and $\epsilon$.

We proceed to state the formal claims associated with steps 1 through 5.

\section{Step 1: The Surrogate Distributions.}

Lemma 5.3 (HARTLINe ET AL. 2011). If bidder $j$ plays $M^{\prime}$ truthfully, then the distribution of the surrogate selected for bidder $j$ is exactly $D_{j}$.

Proof. Imagine sampling replicas and surrogates for bidder $j$ in the following way instead. First, sample the $r$ types for the left-hand side of the bipartite graph i.i.d. from $D_{j}^{+}$and the $r$ types for the right-hand side i.i.d. from $D_{j}$. Then, find the max-weight matching between types, completing it by randomly adding edges from unmatched nodes on the left to unmatched nodes on the right to form a perfect matching. Then, declare all of the $r$ right-hand types surrogates, randomly select one of the left-hand types to be bidder $j$, and declare the remaining $r-1$ as replicas. Note that sampling in this order yields the correct distribution of replicas, surrogates, and bidder $j$, as long as bidder $j$ reports truthfully. Furthermore, it is clear now that the distribution of the surrogate selected for bidder $j$ after sampling in this order is exactly $D_{j}$ : once the matching is fixed, we simply pick a random left-hand type and output its partner. So, essentially, we are drawing $r$ i.i.d. samples from $D_{j}$ and selecting one at random. Clearly this is the same distribution as $D_{j}$.

\section{Step 2: Bayesian Incentive Compatibility.}

Corollary 5.4 (Hartline et Al. 2011). $M^{\prime}$ is BIC.

Proof. Fix any bidder $j$ and assume all others report truthfully. By Lemma 5.3, the distribution of all other surrogates matches $D_{-j}$ exactly, so the weight of the edge between each replica (and bidder $j$ ) and each surrogate correctly computes the value of that replica for being represented by that surrogate in $M$. As bidder $j$ is just participating in a truthful VCG mechanism against the 
replicas for the surrogates, and all values are computed correctly (conditioned on other buyers telling the truth), $M^{\prime}$ is BIC.

Step 3: A Good Matching Implies High Revenue.

Proposition 5.5 (DAskalakis AND Weinberg 2012). Conditioning on right-hand types (surrogates) $\left\{s_{k}\right\}_{k \in[r]}$ and left-hand types (replicas plus bidder $j$ ) $\left\{r_{k}\right\}_{k \in[r]}$, the expected payment of bidder $j$ is at least

$$
\sum_{k \text { s.t. } s_{k}} \sum_{\text {is matched in } V C G} p_{j}^{\epsilon}\left(s_{k}\right) / r .
$$

Proof. Conditioned on the left and right-hand types being sampled, but having not yet decided which left-hand type is bidder $j$, the surrogate matched to bidder $j$ is just a random surrogate. So each surrogate $s_{k}$ is matched to bidder $j$ with probability $1 / r$. Furthermore, bidder $j$ will pay the price $p_{j}^{\epsilon}\left(s_{k}\right)$ whenever his matched surrogate was selected by VCG (and not the random edges afterward). Therefore, bidder $j$ pays at least $\sum_{\left\{k \mid s_{k} \text { is matched in } V C G\right\}} p_{j}^{\epsilon}\left(s_{k}\right) / r$. The reason this is not tight is because it does not count the additional payments of the VCG mechanism.

Step 4: Existence of a Near-Perfect Matching. Our next goal is to show that there exists a nearperfect matching that only matches replicas and surrogates that are "close." For any $\gamma>0$ and two types $v$ and $v^{\prime}$ drawn from $D_{j}$, we say that $v$ and $v^{\prime}$ are $\gamma$-equivalent if for all $S \subseteq[n]$, there exists an integer $z(S)$ such that $\left\{v(S), v^{\prime}(S)\right\} \subseteq\left[(1+\gamma)^{z(S)},(1+\gamma)^{z(S)+1}\right)$. It's easy to see that this defines an equivalence relation. For a type $r_{j}$ drawn from $D_{j}^{+}$, let $r_{j}^{\prime}$ denote it's couple drawn from $D_{j}$. We say that two types drawn from $D_{j}^{+}$are $\gamma$-equivalent if their couples are $\gamma$-equivalent, and that $r_{j}$ drawn from $D_{j}^{+}$is $\gamma$-equivalent to $s_{j}$ drawn from $D_{j}$ if $r_{j}^{\prime}$ and $s_{j}$ are $\gamma$-equivalent (basically, we are putting replicas in equivalence classes based on their couples).

The following lemma from Hartline et al. (2011) bounds the number of unmatched surrogates:

Lemma 5.6 (Lemma 3.7 in HARTLINE ET AL. (2011)). The expected number of unmatched surrogates in a maximal matching that only matches equivalent replicas and surrogates when types are split into at most $\beta$ possible equivalence classes is at most $O(\sqrt{\beta r})$.

In the next lemma, we use Lemma 5.6 to lower-bound the revenue obtained from the matched replicas.

Lemma 5.7 (Implicit in Hartline et Al. (2011)). For any $\gamma>0$, let $W$ denote any maximal matching of replicas to surrogates (for all bidders) that only matches $\gamma$-equivalent types. Then, as $r \rightarrow \infty$, we get $\mathrm{E}\left[\sum_{j} \sum_{\left\{k \mid s_{j k} \text { is matched in } W\right\}} p_{j}^{\epsilon}\left(s_{j k}\right)\right] \geq(1-\gamma) \operatorname{REV}\left(M^{\epsilon}\right)$.

Proof. At a high level, the proof is straightforward: for a fixed equivalence class, the distribution of the number of replicas and surrogates in that class is the same. So as we take more and more i.i.d. samples, the number of each concentrates very tightly around its expectation, so not many types are unmatched. Showing this formally is somewhat technical.

Let's focus on a specific equivalence class $C$ for a fixed bidder $j$. There is some probability $q_{C}$ that a type drawn from $D_{j}$ lands in class $C$. Let $\operatorname{Rev}^{q}\left(M^{\epsilon}\right)$ denote the revenue obtained by $M^{\epsilon}$ only counting payments by types in an equivalence class $C$ such that $q_{C} \geq q$ and $v([n]) \leq 1 / q$ for all $v \in$ $C$. It's clear that $\lim _{q \rightarrow 0} \operatorname{ReV}^{q}\left(M^{\epsilon}\right)=\operatorname{Rev}\left(M^{\epsilon}\right)$, as the revenue obtained from equivalence classes with $q_{C}=0$ is exactly 0 and the revenue obtained from all other equivalence classes is eventually counted for sufficiently small $q$. So we can pick a $q>0$ such that $\operatorname{ReV}^{q}\left(M^{\epsilon}\right) \geq(1-\gamma / 2) \operatorname{Rev}\left(M^{\epsilon}\right)$. Note now that there can only be finitely many equivalence classes counted toward $\operatorname{Rev}^{q}\left(M^{\epsilon}\right)$ (in particular, at most $1 / q$ per bidder), and that the maximum payment by a type in any such equivalence class is at most $1 / q$ (by individual rationality). 
So now the probability that a surrogate or replica is sampled to be in a counted equivalence class is at least $q$ (there must be at least one such equivalence class) and there are at most $1 / q$ equivalence classes. So we may apply Lemma 5.6 to see that for a single bidder, the expected number of unmatched surrogates from equivalence classes that count is at most $O(\sqrt{r / q})$. As each such surrogate pays at most $1 / q$, the total revenue lost in expectation from unmatched surrogates in equivalence classes that count is at most $O\left(1 / \sqrt{r q^{3}}\right)$ (due to Proposition 5.5). Summing up across all bidders, the revenue lost is at most $O\left(\mathrm{~m} / \sqrt{r q^{3}}\right)$. In addition, the total revenue lost in expectation from unmatched surrogates across all bidders from equivalence classes that didn't count is clearly at most $(\gamma / 2) \operatorname{Rev}\left(M^{\epsilon}\right)$ by choice of $q$. So the total revenue lost from unmatched surrogates in this matching is at most $(\gamma / 2) \operatorname{Rev}\left(M^{\epsilon}\right)+O\left(m / \sqrt{r q^{3}}\right)$. As $r \rightarrow \infty$, the second term approaches zero, completing the proof.

Step 5: The VCG Matching Is Almost as Good. Combining Proposition 5.5 and Lemma 5.7 says the following: if only this nearly perfect matching was the one selected by VCG, then we would know that $\operatorname{REV}^{M^{\prime}}(D)$ was good. But for all we know, VCG may choose to leave many surrogates unmatched if it helps improve the welfare of the replicas. The key is to show that not many surrogates can be unmatched, due to the rebates.

Let $V$ denote the VCG matching and $W$ denote the matching guaranteed by Lemma 5.7. Then there is a disjoint set of augmenting paths and cycles that transform $W$ into $V$. As $V$ is a maxweight matching, all of these augmenting paths and cycles must have non-negative weight. It is easy to see that augmenting cycles do not change the set of matched surrogates, and therefore do not affect the revenue. We therefore want to study augmenting paths that unmatch a surrogate.

If an augmenting path unmatches $s_{j k}$, then no replica is receiving the rebate awarded to $s_{j k}$ any more. Because VCG is choosing the max-weight matching, it must be the case that the benefit of switching every other edge along the path outweighs the cost of losing the rebate awarded to $s_{j k}$. This is where we make use of the fact that each replica is matched to a surrogate that is nearly identical to them, except for an additive $\delta_{j k}(\cdot)$. Because $M$ is BIC, we can bound the expected gain of switching a replica who is matched to a nearly identical surrogate to any other surrogate using $\delta(\cdot)$. Therefore, each surrogate that gets unmatched by an augmenting path "claims" many replicas to be in its augmenting path. The argument shows that in fact it takes several replicas to make a positive weight augmenting path, and therefore not many surrogates can be unmatched.

Lemma 5.8 (Ideas from Daskalakis ANd Weinberg (2012)). If $U_{j}$ denotes the set of surrogates that are matched in $W$ but not $V$, and $T_{j}$ denotes the set of surrogates matched in $V$ but not $W$, (for bidder $j)$, then $\mathrm{E}\left[\frac{1}{r} \sum_{j} \sum_{s \in U_{j}} p_{j}(s)-\sum_{s \in T_{j}} p_{j}(s)\right] \leq \operatorname{VAL}(\delta) / \epsilon$.

Proof. Consider any augmenting path that unmatches a surrogate $s$ in $W$ and (possibly) matches a new surrogate $s^{\prime}$. For simplicity of notation, if no new surrogate is matched, we let $s^{\prime}$ denote a null type that receives no items and pays no price to $M$. We break the contribution of edges in this path into two parts, the first coming just from the rebates awarded to the surrogates and the second coming from the allocation and original price paid. It is easy to see that the contribution of rebates to the weight of the augmenting path is exactly $\epsilon p_{j}(s)-\epsilon p_{j}\left(s^{\prime}\right)$. Now we analyze the weight of the path coming from the second part. We can compute the weight by summing over all replicas $r_{j}$ in the path of their utility for their new surrogate minus their utility for their old surrogate. Note that any augmenting path that unmatches a surrogate cannot possibly add an edge to a replica who was unmatched in $W$. Since $M$ is BIC, for any replica $r_{j}$ that was matched to $s_{j}$ in $W$ and moved to $s_{j}^{\prime}$ in $V$, we have

$$
s_{j}\left(\phi_{j}\left(s_{j}\right)\right)-p_{j}\left(s_{j}\right) \geq s_{j}\left(\phi_{j}\left(s_{j}^{\prime}\right)\right)-p_{j}\left(s_{j}\right) .
$$


Using the fact that $r_{j}$ and $s_{j}$ are $\gamma$-equivalent, we get

$$
(1+\gamma) r_{j}\left(\phi_{j}\left(s_{j}\right)\right)-p_{j}\left(s_{j}\right) \geq(1-\gamma) r_{j}\left(\phi_{j}\left(s_{j}^{\prime}\right)\right)-\delta_{j}\left(\phi_{j}\left(s_{j}^{\prime}\right)\right)-p_{j}\left(s_{j}\right) .
$$

And rearranging terms yields

$$
r_{j}\left(\phi_{j}\left(s_{j}^{\prime}\right)\right)-p_{j}\left(s_{j}\right)-r_{j}\left(\phi_{j}\left(s_{j}\right)\right)+p_{j}\left(s_{j}\right) \leq \gamma r_{j}\left(\phi_{j}\left(s_{j}\right)\right)+\gamma r_{j}\left(\phi_{j}\left(s_{j}^{\prime}\right)\right)+\delta_{j}\left(\phi_{j}\left(s_{j}^{\prime}\right)\right) .
$$

Note that the left-hand side is exactly the increase in utility as we move $r_{j}$ from $s_{j}$ to $s_{j}^{\prime}$. So now we know that the total increase in utility from moving all replicas across all bidders must outweigh the total decrease caused by unmatching surrogates. We therefore get

$$
\mathrm{E}\left[\frac{1}{r} \sum_{j} \sum_{s \in U_{j}} p_{j}(s)-\sum_{s \in T_{j}} p_{j}(s)\right] \leq \mathrm{E}\left[\frac{1}{r} \sum_{j} \sum_{r_{j}} \gamma r_{j}\left(\phi_{j}\left(s_{j}\right)\right)+\gamma r_{j}\left(\phi_{j}\left(s_{j}^{\prime}\right)\right)+\delta_{j}\left(\phi_{j}\left(s_{j}^{\prime}\right)\right)\right] .
$$

Consider now the following allocation algorithm (not a mechanism, and certainly not truthful). When bidder $j$ reports $r_{j}$, the algorithm selects the allocation output by $M$ on input $\left(s_{1}, \ldots, s_{m}\right)$. This is clearly a feasible allocation algorithm, and it's also clear that the term $\mathrm{E}\left[\frac{1}{r} \sum_{j} \sum_{r_{j}} r_{j}\left(\phi_{j}\left(s_{j}\right)\right)\right]$ exactly computes the expected welfare achieved by this algorithm when the type of buyer $j$ is drawn from $D_{j}$. We can similarly define an allocation algorithm that replaces $r_{j}$ with $s_{j}^{\prime}$, and one that samples $\delta_{j} \leftarrow D_{j}^{+}-D_{j}$ and replaces $\delta_{j}$ with $s_{j}^{\prime}$. Now that we have concrete allocation algorithms that match the terms on the right-hand side exactly, we can bound it as

$$
\mathrm{E}\left[\frac{1}{r} \sum_{j} \sum_{r_{j}} \gamma r_{j}\left(\phi_{j}\left(s_{j}\right)\right)+\gamma r_{j}\left(\phi_{j}\left(s_{j}^{\prime}\right)\right)+\delta_{j}\left(\phi_{j}\left(s_{j}^{\prime}\right)\right)\right] \leq 2 \gamma \operatorname{VAL}(D)+\operatorname{VAL}(\delta) .
$$

Lastly, because this claim holds for all $\gamma>0$, we may let $\gamma \rightarrow 0$ and the right-hand bound becomes just $\operatorname{VAL}(\delta)$. Some care must be taken if $\operatorname{VAL}(D)=\infty$, but similar tricks to those used in the proof of Lemma 5.7 suffice. Essentially, one can take an increasing limit of truncations of $D$, calling them $D^{C}$ (the same distribution $D$ but replacing $v(\cdot)$ with the 0 -function if $\left.v([n])>C\right)$. Clearly all $\operatorname{VAL}\left(D^{C}\right)$ is finite for all $C$, and clearly $\lim _{C \rightarrow \infty} \operatorname{ReV}^{M}\left(D^{C}\right)=\operatorname{ReV}^{M}(D)$ for any mechanism $M$. So one can use the above analysis on the bounded distributions $D^{C}$ and take a limit.

With the lemma above, our proof is complete. There is a high-cardinality matching $W$ that provides revenue exactly $\operatorname{ReV}^{M}(D)$, if only it were chosen by VCG. But VCG may choose a different matching, and we may lose some revenue. The lemma bounds how much revenue can be lost, and provides the bound in the theorem.

\section{ACKNOWLEDGMENT}

We would like to thank Moshe Babaioff, Hu Fu, Nicole Immorlica, and Brendan Lucier for numerous suggestions and helpful discussions.

\section{REFERENCES}

Moshe Babaioff, Nicole Immorlica, Brendan Lucier, and S. Matthew Weinberg. 2014. A simple and approximately optimal mechanism for an additive buyer. In Proceedings of the IEEE Foundations of Computer Science (FOCS'14).

MohammadHossein Bateni, Sina Dehghani, MohammadTaghi Hajiaghayi, and Saeed Seddighin. 2015. Revenue maximization for selling multiple correlated items. In Algorithms-ESA 2015, 23rd Annual European Symposium, Patras, Greece, September 14-16, 2015, Proceedings. 95-105. DOI : http://dx.doi.org/10.1007/978-3-662-48350-3_9

Xiaohui Bei and Zhiyi Huang. 2011. Bayesian incentive compatibility via fractional assignments. In Proceedings of the 22nd Annual ACM-SIAM Symposium on Discrete Algorithms (SODA'11).

Patrick Briest, Shuchi Chawla, Robert Kleinberg, and S. Matthew Weinberg. 2010. Pricing randomized allocations. In Proceedings of the ACM-SIAM Symposium on Discrete Algorithms. 585-597. 
Yang Cai, Constantinos Daskalakis, and S. Matthew Weinberg. 2013. Understanding incentives: Mechanism design becomes algorithm design. In Proceedings of the 54th Annual IEEE Symposium on Foundations of Computer Science (FOCS'13). http://arxiv.org/pdf/1305.4002v1.pdf.

Yang Cai, Nikhil R. Devanur, and S. Matthew Weinberg. 2016. A duality based unified approach to Bayesian mechanism design. In Proceedings of the 48th Annual ACM SIGACT Symposium on Theory of Computing, STOC 2016, Cambridge, MA, June 18-21, 2016. 926-939. DOI : http://dx.doi.org/10.1145/2897518.2897645

Yang Cai and Zhiyi Huang. 2013. Simple and nearly optimal multi-item auctions. In Proceedings of the 24th Annual ACMSIAM Symposium on Discrete Algorithms (SODA'13).

Yang Cai and Mingfei Zhao. 2017. Simple mechanisms for subadditive buyers via duality. In Proceedings of the 49th Annual ACM Symposium on Theory of Computing (STOC'17).

Shuchi Chawla, Jason D. Hartline, and Robert Kleinberg. 2007. Algorithmic pricing via virtual valuations. In Proceedings of the 8th ACM Conference on Electronic Commerce (EC'07). 243-251.

Shuchi Chawla, Jason D. Hartline, David L. Malec, and Balasubramanian Sivan. 2010a. Multi-parameter mechanism design and sequential posted pricing. In Proceedings of the 42nd ACM Symposium on Theory of Computing (STOC'10).

Shuchi Chawla, David L. Malec, and Balasubramanian Sivan. 2010b. The power of randomness in bayesian optimal mechanism design. In Proceedings of the 11th ACM Conference on Electronic Commerce (EC'10), Cambridge, Massachusetts, fune 7-11, 2010. 149-158. DOI : http://dx.doi.org/10.1145/1807342.1807366

Shuchi Chawla and J. Benjamin Miller. 2016. Mechanism design for subadditive agents via an ex ante relaxation. In Proceedings of the 2016 ACM Conference on Economics and Computation (EC'16), Maastricht, The Netherlands, fuly 24-28, 2016. 579-596. DOI : http://dx.doi.org/10.1145/2940716.2940756

Xi Chen, Ilias Diakonikolas, Dimitris Paparas, Xiaorui Sun, and Mihalis Yannakakis. 2014. The complexity of optimal multidimensional pricing. In Proceedings of the 25th Annual ACM-SIAM Symposium on Discrete Algorithms (SODA'14).

Constantinos Daskalakis, Alan Deckelbaum, and Christos Tzamos. 2014. The complexity of optimal mechanism design. In Proceedings of the 25th Annual ACM-SIAM Symposium on Discrete Algorithms (SODA'14).

Constantinos Daskalakis and S. Matthew Weinberg. 2012. Symmetries and optimal multi-dimensional mechanism design. In Proceedings of the 13th ACM Conference on Electronic Commerce (EC'12).

Alon Eden, Michal Feldman, Ophir Friedler, Inbal Talgam-Cohen, and S. Matthew Weinberg. 2017. A simple and approximately optimal mechanism for a buyer with complements. In Proceedings of the 18th Annual ACM Conference on Economics and Computation (EC'17).

Michal Feldman, Nick Gravin, and Brendan Lucier. 2015. Combinatorial auctions via posted prices. In Proceedings of the 26th Annual ACM-SIAM Symposium on Discrete Algorithms (SODA'15). Society for Industrial and Applied Mathematics, Philadelphia, PA, 123-135. http://dl.acm.org/citation.cfm?id=2722129.2722139

Sergiu Hart and Noam Nisan. 2012. Approximate revenue maximization with multiple items. In Proceedings of the 13th ACM Conference on Electronic Commerce (EC'12). 656-656.

Sergiu Hart and Noam Nisan. 2013. The menu-size complexity of auctions. In Proceedings of the 14th ACM Conference on Electronic Commerce (EC'13). 565-566.

Sergiu Hart and Philip J. Reny. 2012. Maximal Revenue with Multiple Goods: Nonmonotonicity and Other Observations. Discussion Paper Series dp630. The Center for the Study of Rationality, Hebrew University, Jerusalem.

Jason D. Hartline. 2011. Approximation and Mechanism Design. http://jasonhartline.com/MDnA/.

Jason D. Hartline, Robert Kleinberg, and Azarakhsh Malekian. 2011. Bayesian incentive compatibility via matchings. In The 22nd Annual ACM-SIAM Symposium on Discrete Algorithms (SODA'11).

Robert Kleinberg and S. Matthew Weinberg. 2012. Matroid prophet inequalities. In The 44th Annual ACM Symposium on Theory of Computing (STOC'12).

Xinye Li and Andrew Chi-Chih Yao. 2013. On revenue maximization for selling multiple independently distributed items. Proceedings of the National Academy of Sciences 110, 28 (2013), 11232-11237.

Will Ma and David Simchi-Levi. 2015. Reaping the benefits of bundling under high production costs. CoRR abs/1512.02300 (2015). http://arxiv.org/abs/1512.02300.

Roger B. Myerson. 1981. Optimal auction design. Mathematics of Operations Research 6, 1 (1981), 58-73.

Christos H. Papadimitriou, George Pierrakos, Christos-Alexandros Psomas, and Aviad Rubinstein. 2016. On the complexity of dynamic mechanism design. In Proceedings of the 27th Annual ACM-SIAM Symposium on Discrete Algorithms (SODA'16), Arlington, VA, Fanuary 10-12, 2016. 1458-1475. DOI : http://dx.doi.org/10.1137/1.9781611974331.ch100

Gregory Pavlov. 2011. A property of solutions to linear monopoly problems. B. E. fournal of Theoretical Economics 11, 1 (2011), Article 4.

John Riley and Richard Zeckhauser. 1983. Optimal selling strategies: When to haggle, when to hold firm. Quarterly fournal of Economics 98, 2 (1983), 267-289.

Aviad Rubinstein. 2016. On the computational complexity of optimal simple mechanisms. In Proceedings of the 2016 ACM Conference on Innovations in Theoretical Computer Science, Cambridge, MA, January 14-16, 2016. 21-28. DOI : http://dx. doi.org/10.1145/2840728.2840736 
Aviad Rubinstein and Sahil Singla. 2017. Combinatorial prophet inequalities. In Proceedings of the 28th Annual ACMSIAM Symposium on Discrete Algorithms (SODA'17), Barcelona, Spain, Hotel Porta Fira, Fanuary 16-19. 1671-1687. DOI : http://dx.doi.org/10.1137/1.9781611974782.110

Gideon Schechtman. 2003. Concentration, results and applications. Handbook of the Geometry of Banach Spaces, W. B. Johnson and J. Lindenstrauss (Eds.). Vol. 2. North Holland.

John Thanassoulis. 2004. Haggling over substitutes. Journal of Economic Theory 117 (2004), 217-245.

Andrew Chi-Chih Yao. 2015. An $n$-to-1 bidder reduction for multi-item auctions and its applications. In Proceedings of the 26th Annual ACM-SIAM Symposium on Discrete Algorithms (SODA'15)

Received February 2016; revised February 2017; accepted May 2017 